\title{
Realistic Modelling of Irregular Slabs under Extreme Loading
}

\section{Stanyslav Grosman}

s.grosman15@imperial.ac.uk

Department of Civil and Environmental Engineering, Imperial College, London, SW7 2AZ

http://www.imperial.ac.uk/csm

ORCID: 0000-0003-4474-7838

Bassam A. Izzuddin, Professor

b.izzuddin@imperial.ac.uk

Department of Civil and Environmental Engineering, Imperial College, London, SW7 2AZ

http://www.imperial.ac.uk/csm

ORCID: 0000-0001-5746-463X 


\begin{abstract}
This paper presents a new triangular flat shell element for composite and reinforced concrete slabs of complex planar configuration subjected to extreme loading. The element is developed within a co-rotational framework, and it incorporates the effects of geometric as well as material nonlinearities. To improve the approximation of the solution and deal with shear locking, additional hierarchic parameters are introduced within the local system of the element, providing an option to activate higher-order quadratic approximation for the element shape functions. The element formulation allows for composite action between different layers under the assumption of perfect bond between the slab concrete material, the reinforcement layers and the steel deck for composite slabs. To account for floor slabs of irregular geometric configurations, due allowance is made for uniaxial reinforcement to be oriented arbitrarily within the slab plane. Furthermore, an algorithm for automatic rebar orientation is developed for irregular slabs with realistic reinforcement distributions. The paper briefly describes the element formulation followed by several numerical verification examples. The applicability of the element to modelling concrete slabs is demonstrated via several validation studies against existing experimental results. The versatility of the element is further exemplified with a realistic large-scale floor slab model subjected to extreme loading scenarios. It is shown that the developed element provides a good balance between accuracy and efficiency in the modelling of irregular floor slabs subject to extreme loading conditions.
\end{abstract}

Keywords: Computational mechanics, Concrete structures; Mathematical modelling; Slabs \& plates; Shells 


\section{Introduction}

Over recent years, the tendency of modern day architects to use singular and non-repeatable shapes for building structures has been gaining popularity. There are numerous types of such irregularities: different floor shapes along the structure height, inclination of a building vertical axis, inclusion of twist into the structures general shape, etc. These unique features make such buildings aesthetically pleasing and financially attractive.

To ensure that such irregular buildings meet structural safety requirements, detailed numerical analysis is often necessary. Some practical considerations for analysis of irregular structures are outlined by Shin, Kang and Grossman (2009). Typical simplified approaches can reduce the computational demand but at a cost of significant loss of accuracy as the system response becomes more complex and nonlinear in nature. Nowadays, it has become important to account for extreme loading, such as blast and earthquakes, when analysing response of high profile buildings. The most commonly employed strategy to conduct such an assessment is through the use of finite element analysis (FEA). With this approach, the most complex and computationally demanding part of the numerical modelling process is related to the numerical representation of $2 \mathrm{D}$ continuous bodies such as slabs and walls. For irregular structures, the use of quadrilateral elements becomes cumbersome even with the most sophisticated mesh generators, leading to distorted element shapes that can adversely affect accuracy, particularly in nonlinear analysis under extreme loading. As such the most robust way of discretising irregular 2D continuum structures is based around triangular element meshes.

Shell elements have been the subject of extensive research over several recent decades. Many different formulations capable of representing nonlinear behaviour of shells with different geometric configurations have been proposed for both triangular (Lee, Lee and Bathe, 2014) and quadrilateral (Dvorkin and Bathe, 1984; Swamy Naidu and Sateesh, 2015; Ko, Lee and Bathe, 2017) elements. The most common formulations are based around Reissner-Mindlin plate theory with modifications to the shear strain fields to alleviate locking problems. The development and application of shell elements for nonlinear analysis of concrete slabs and walls has also been an active area of research. Recently Song et al. (2002) proposed a layered serendipity shell element capable of capturing the response of concrete structures under cyclic loading conditions, where the element formulation was enhanced via the inclusion of an extra pressure parameter which can assist with convergence. The drawback of this approach is that this modification is incapable of dealing with convergence issues caused by such instabilities as snapback and snap-through, which can be observed for some concrete structures (Crisfield, 2000b), besides the introduction of an additional parameter to the element degrees of freedom (DOF). A similar layered approach was adopted by Zhang and Zhu (2010) for a fibre reinforced concrete shell element, where they introduced a different approach for dealing with locking problems by incorporating Timoshenko composite beam functions into the formulation. This approach, however, comes at the cost of loss of generality, and as such the described element is restricted to rectangular shapes. A different approach for the 
assessment of concrete shells was adopted by Liu and Teng (2008), where instead of adopting a layered approach for the 9-noded shell element, with material strains and stresses evaluated for individual layers, the authors assumed a predefined stress distribution through the element and formulated the element response based on the generalised cross-sectional approach. This simplified approach allowed a good prediction of the response in bending dominated problems, though the accuracy is reduced for slabs involving coupled bending/membrane behaviour. This drop in accuracy can be explained by the simplified material model employed and by the neglect of geometric nonlinearity in the element formulation.

To address the need for effective nonlinear analysis of reinforced concrete (RC) floor slabs and shear walls, for particular application to irregular structures, a new 2D shell element is proposed. This element combines the computational efficiency of three-noded elements, with modelling accuracy achieved by incorporation of hierarchic quadratic and cubic terms into the shape functions. The developed element is also capable of accounting for both geometric and material nonlinearities.

In contrast with a rectangular shell element for composite slabs developed by Izzuddin et al. (2004), the proposed element allows for arbitrary slab shapes and rebar orientations. These properties allow the proposed element to be used in modelling irregular floor slabs under extreme conditions, including those due to fire, blast and earthquake loading. In addition, the element is applicable to the modelling of concrete slabs strengthened with strips of composite material.

The paper presents the formulation of the proposed triangular element within a local reference system, including the consideration of hierarchic shape functions for improved accuracy and the treatment of arbitrary rebar orientation for irregular floor slabs. Subsequently, the incorporation of the developed triangular element within a co-rotational approach (Izzuddin, 2005; Izzuddin and Liang, 2016) is discussed, which enables the consideration of geometric nonlinearity including large displacements. The proposed triangular shell element has been implemented within the nonlinear structural analysis program ADAPTIC (Izzuddin, 1991), which is used in this paper to provide verification tests for the element. Several verification and validation examples are presented to demonstrate the effectiveness and accuracy of the proposed element for geometric and material nonlinear analysis of reinforced concrete slabs. A final example is also provided to illustrate one of the applications of the proposed element, which relates to a large scale irregular structure subjected to the case of sudden column loss, as typically considered for the assessment of structural robustness.

\section{Kinematics of Triangular Shell Element}

The proposed shell element for RC and composite floor slabs is formulated within a local co-rotational framework for large-displacement analysis under following assumptions:

1. the concrete slab depth and the thickness of the steel deck in a composite slab are uniform; 
2. the rebar layout within a single element can be approximated with either parallel or orthogonal layers;

3. the reinforcement response is uniaxial, in the direction of the rebar orientation;

4. perfect bond exists between concrete and both the reinforcement and the steel deck; and

5. direct out-of-plane stresses in the concrete are negligible.

The proposed kinematic description, representing the Reissner-Mindlin hypothesis for thin to moderately thick plates, is formulated with reference to a local Cartesian coordinates system, with the origin located at node 1 of the element, and with the local $\mathrm{X}$-axis passing through node 2, as depicted in Figure 1. In addition, natural area coordinates are employed for a simplified derivation of the element shape functions.

The kinematic dependency that defines the displacement fields $(u, v, w)$ in terms of area coordinates $\left(\lambda_{1}, \lambda_{2}, \lambda_{3}\right)$, according to the adopted Reissner-Mindlin hypothesis (Zienkiewicz and Taylor, 2000b), is related to the reference planar displacements $\left(u_{p}, v_{p}, w_{p}\right)$ and rotations of the shell normal $\left(\theta_{x}, \theta_{y}\right)$ by the following expressions:

$$
\left\{\begin{array}{l}
u\left(\lambda_{1}, \lambda_{2}, \lambda_{3}\right)=u_{p}\left(\lambda_{1}, \lambda_{2}, \lambda_{3}\right)-z \cdot \theta_{x}\left(\lambda_{1}, \lambda_{2}, \lambda_{3}\right) \\
v\left(\lambda_{1}, \lambda_{2}, \lambda_{3}\right)=v_{p}\left(\lambda_{1}, \lambda_{2}, \lambda_{3}\right)-z \cdot \theta_{y}\left(\lambda_{1}, \lambda_{2}, \lambda_{3}\right) \\
w\left(\lambda_{1}, \lambda_{2}, \lambda_{3}\right)=w_{p}\left(\lambda_{1}, \lambda_{2}, \lambda_{3}\right)
\end{array}\right.
$$

where area coordinates are related to local element coordinates $(\xi, \eta)$ as follows:

$$
\left\{\begin{array}{l}
\lambda_{1}=1-\lambda_{2}-\lambda_{3} ; \\
\lambda_{2}=\frac{x \cdot y_{3}-y \cdot x_{3}}{A_{2}} \\
\lambda_{3}=\frac{y}{y_{3}} ;
\end{array}\right.
$$

with $A_{2}$ standing for twice the element area.

The five components of the considered strain tensor can be established from (1) by derivation with respect to the local coordinates: 


$$
\left\{\begin{array}{l}
\varepsilon_{x}=\frac{\partial u_{p}}{\partial x}-z \cdot \frac{\partial \theta_{x}}{\partial x} \\
\varepsilon_{y}=\frac{\partial v_{p}}{\partial y}-z \cdot \frac{\partial \theta_{y}}{\partial y} \\
\gamma_{x y}=\frac{\partial u_{p}}{\partial y}+\frac{\partial v_{p}}{\partial x}-z \cdot\left(\frac{\partial \theta_{y}}{\partial x}+\frac{\partial \theta_{x}}{\partial y}\right) \\
\gamma_{x z}=-\theta_{x}+\frac{\partial w}{\partial x} \\
\gamma_{z y}=-\theta_{y}+\frac{\partial w}{\partial y}
\end{array}\right.
$$

where derivatives of reference planar deformation fields $\left(\psi \equiv\left[u_{p}, v_{p}, w_{p}, \theta_{x}, \theta_{y}\right]\right)$ can be obtained in the local system by the means of chain rule differentiation from the respective field derivatives in the area coordinate system:

$$
\begin{aligned}
& \frac{\partial \psi}{\partial x}=\sum_{j=1}^{3} \frac{\partial \psi}{\partial \lambda_{j}} \cdot \frac{\partial \lambda_{j}}{\partial x} \\
& \frac{\partial \psi}{\partial y}=\sum_{j=1}^{3} \frac{\partial \psi}{\partial \lambda_{j}} \cdot \frac{\partial \lambda_{j}}{\partial y}
\end{aligned}
$$

To account for the influence of reinforcing material on the response of the RC/composite slab element, the system response is enriched with the contribution from each of the considered layers, including rebars and steel deck. Uniaxial strains in the rebars can be obtained as a sum of projections of element planar strains on the rebar orientation defined by the angle $(\alpha)$ between the rebar and the element side 1-2 (see Figure 1):

$$
\varepsilon_{s}=\varepsilon_{x} \cdot \cos (\alpha)^{2}+\varepsilon_{y} \cdot \sin (\alpha)^{2}+\gamma_{x y} \cdot \sin (\alpha) \cdot \cos (\alpha)
$$

The steel deck strain components $\left(\varepsilon_{x}, \varepsilon_{y}, \gamma_{x y}\right)$ are directly obtained from (3), by considering only the very bottom layer of the slab with $\left(z=-\frac{h}{2}\right)$

To allow for unstructured mesh definitions, an automatic procedure for the determination of rebar orientation angle $(\alpha)$ is considered based on two alternative approaches. The first alternative utilises a predefined vector in the global coordinate 
system which is projected on the element plane to establish one of the directions of reinforcement, with the other direction being orthogonal. The second alternative utilises a point (A) in global coordinates whose projection (A') onto the element plane (P) along with the element centre $\left(\mathrm{C}_{\mathrm{i}}\right)$ defines a vector $\left(v_{i}\right)$ that is orthogonal to the reinforcement direction (Figure 2). This latter alternative can be employed for the straightforward specification of radial rebars and for a more general definition of rebar orientation in silos and more complex structures (Calavera, 2012; Schofield, 2012) modelled with flat shell elements.

\section{Local Element Response}

The kinematic description in the previous section is presented as a relationship between the element strains and the planar displacements $\left(u_{p}, v_{p}, w_{p}\right)$ and the normal rotations $\left(\theta_{x}, \theta_{y}\right)$. These fields are discretised in terms of basic and hierarchic DOF using polynomial shape functions.

Employing triangular elements with quadratic shape functions results in the addition of DOF parameters to the system while using basic linear shape functions results in loss of accuracy. To address these issues, selective enrichment with hierarchic shape functions (Zienkiewicz and Taylor, 2000a) is employed. Hierarchic DOFs represent coefficients of the hierarchic shape functions, the physical meaning of which is incremental on top of the basic shape functions, as illustrated in Figure 3.

As such the general form for the local element displacement field $\boldsymbol{\psi}$ is as follows:

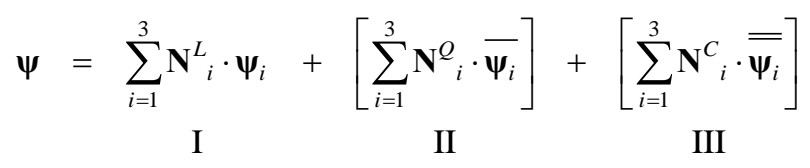

III

(6)

where $\left(\boldsymbol{\psi}_{i}, \overline{\boldsymbol{\psi}_{i}}, \overline{\overline{\boldsymbol{\psi}_{i}}}\right)$ represent the nodal DOF (I), the hierarchic DOF related to quadratic shape functions (II), and the hierarchic DOF related to cubic shape functions (III), respectively.

The linear nodal shape functions as well as the quadratic and cubic hierarchic shape functions are conveniently defined in the area coordinate system as follows:

$$
\mathbf{N}_{i}^{L}=\left[\begin{array}{l}
\lambda_{1} \\
\lambda_{2} \\
\lambda_{3}
\end{array}\right] ; \mathbf{N}^{Q}{ }_{i}=4 \cdot\left[\begin{array}{c}
\lambda_{1} \cdot \lambda_{2} \\
\lambda_{2} \cdot \lambda_{3} \\
\lambda_{3} \cdot \lambda_{1}
\end{array}\right] ; \mathbf{N}_{i}^{C}=6 \cdot \sqrt{3} \cdot\left[\begin{array}{c}
\lambda_{1} \cdot \lambda_{2} \cdot\left(\lambda_{2}-\lambda_{1}\right) \\
\lambda_{2} \cdot \lambda_{3} \cdot\left(\lambda_{3}-\lambda_{2}\right) \\
\lambda_{3} \cdot \lambda_{1} \cdot\left(\lambda_{3}-\lambda_{1}\right)
\end{array}\right]
$$


One of the additional benefits of employing hierarchic DOF is that the element can be used in either linear or higherorder form which allows for a more versatile modelling of complex problems. For instance for a section of a structure, where accuracy is not of paramount importance, shape functions can be reduced to their linear form to reduce the number of DOF in the system and as such reduce the computational demand. Another benefit comes from the fact that stiffness matrices associated with hierarchic DOFs tend to be more diagonal (Zienkiewicz and Taylor, 2000a) and as such are more numerically stable, which is very important when dealing with complex nonlinear behaviour of such material as concrete. As a further added benefit, the hierarchic formulation also allows for transverse displacement shape function to be always one order higher than the rotations of the shell normal, which reduces shear locking effects (Hughes and Tezduyar, 1981). A final less obvious benefit that arises from considering a hierarchic formulation for the local element response in a co-rotational framework for large displacement analysis is that the additional hierarchic parameters can be excluded from the computationally demanding co-rotational transformations. This will be discussed further in in the following section.

After each incremental step, element strains for both the concrete slab, steel deck and reinforcement layers can be established based on the equations (3-7). The evaluation is performed at Gauss points defined for the numerical integration of triangular element (Dunavant, 1985; Zienkiewicz and Taylor, 2000a). A robust nonlinear constitutive relationship for concrete (Izzuddin, Tao and Elghazouli, 2004) as well as standard elasto-plastic models for steel are employed to obtain stresses at the considered Gauss points $\left(\boldsymbol{\sigma}^{c}, \boldsymbol{\sigma}^{d}, \boldsymbol{\sigma}^{s_{t}}\right)$. The contributions of the constituent materials to the local resistance forces vector and tangent stiffness matrix are obtained via numerical integration over the element domain for each of the constituent materials:

$$
\mathbf{f}_{e}=\mathbf{f}_{e}^{c}+\left(\sum_{i=1}^{N} \mathbf{f}_{e_{i}}^{s}\right)+\mathbf{f}_{e}^{d}
$$

$$
\mathbf{K}_{e}=\mathbf{K}_{e}^{c}+\left(\sum_{i=1}^{N} \mathbf{K}_{e_{i}}^{s}\right)+\mathbf{K}_{e}^{d}
$$

$$
\mathbf{f}_{e}^{c}=\frac{A_{2} \cdot h}{4} \cdot \sum_{k=1}^{m} \sum_{j=1}^{n} w_{k}^{h} \cdot w_{j}^{p} \cdot\left(\mathbf{B}_{j k}^{c}\right)^{T} \cdot \boldsymbol{\sigma}_{j k}^{c}
$$


$\mathbf{K}_{e}^{c}=\frac{A_{2} \cdot h}{4} \cdot \sum_{k=1}^{m} \sum_{j=1}^{n} w_{k}^{h} \cdot w_{j}^{p} \cdot\left(\mathbf{B}_{j k}^{c}\right)^{T} \cdot \mathbf{D}_{j k}^{c} \cdot \mathbf{B}_{j k}^{c}$

$\mathbf{f}_{e}^{d}=\frac{A_{2} \cdot t_{d}}{4} \cdot \sum_{j=1}^{n} \cdot w_{j}^{p} \cdot\left(\mathbf{B}_{j}^{d}\right)^{T} \cdot \boldsymbol{\sigma}^{d}{ }_{j}$

$\mathbf{K}_{e}^{d}=\frac{A_{2} \cdot t_{d}}{4} \cdot \sum_{j=1}^{n} w_{j}^{p} \cdot\left(\mathbf{B}_{j}^{d}\right)^{T} \cdot \mathbf{D}_{j}^{d} \cdot \mathbf{B}_{j}^{d}$

$\mathbf{f}_{e_{i}}^{s}=\frac{A_{2} \cdot\left(t_{e}\right)_{i}}{4} \cdot \sum_{j=1}^{n} \cdot w_{j}^{p} \cdot\left(\mathbf{B}_{j}^{s}\right)_{i}^{T} \cdot\left(\boldsymbol{\sigma}_{j}^{s}\right)_{i}$

$\mathbf{K}_{e}^{s}=\frac{A_{2} \cdot\left(t_{e}\right)_{i}}{4} \cdot \sum_{j=1}^{n} w_{j}^{p} \cdot\left(\mathbf{B}_{j}^{s}\right)_{i}^{T} \cdot\left(\mathbf{D}_{j}^{s}\right)_{i} \cdot\left(\mathbf{B}_{j}^{s}\right)_{i}$

where $N$ stands for the number of rebar layers, superscripts $(c, d, s)$ refer to the concrete slab, deck and rebar layer components, and $\left(t_{d}, t_{s}\right)$ correspond to thickness of deck and effective thickness of rebar layer, respectively, noting that rebars are considered in terms of a smeared layer thickness. Numerical integration weighting factors $\left(w^{p}, w^{h}\right)$ refer to integration over the plane and slab thickness, respectively, with $(n, m)$ representing the corresponding numbers of Gauss points. Finally, the $\mathbf{B}$ matrices relate the strain components of constituent materials to the local displacements, and these can be readily established form (3-7), while the D terms represent the tangent modulus matrices for the considered material.

It is worth noting that while the above local formulation accounts for material nonlinearity, it excludes local geometric nonlinearity. However, the incorporation of the element within a co-rotational framework enables the direct consideration of geometric nonlinearity, with the influence of local geometric nonlinearity becoming less significant with mesh refinement, as is typically required for modelling complex and irregular floor slab configurations. 


\section{Co-rotational Framework}

As outlined by Belytschko et al. (2000), when a structural system experiences large rotations, linear deformation measures are no longer applicable. To address this issue the concept of rotated stresses and strains was introduced, such quantities referring to a measure of deformation that excludes rigid body rotations. Crisfield (2000a, 2000b) further expanded this concept, taking it from the material point level to the element level. This was accomplished by defining a local reference coordinate system that would follow the deformed element in such a way as to ensure that the element would experience in this system only small translations and rotations. This approach allows the use of elements that are geometrically linear in the local system, with geometric nonlinearity incorporated through transformations between local co-rotational reference system and global coordinate system.

The proposed flat shell is accordingly considered within a co-rotational framework for geometric nonlinear analysis, adopting approaches developed by Izzuddin, (2005) and Izzuddin and Liang (2016). In order to remove the influence of the rigid body rotations from the local element response, a local co-rotational system is established in such a way as to minimise the rotation of the deformed shell element. Two existing approaches are considered for this purpose with the proposed element: bisector and zero-macrospin (Izzuddin, 2005; Izzuddin and Liang, 2016), which provide the required discrete transformations between local and global element parameters.

The bisector approach was initially developed by Izzuddin (2005) for quadrilateral elements and later expanded by Izzuddin and Liang (2016) to triangular elements. The approach places the co-rotated local system in such a way so that the bisector of an internal angle, formed by the centroid and vertices of the undeformed triangle, is coincident with the corresponding bisector for the triangular element in the deformed configuration, as illustrated in Figure 4.

On the other hand, the alternative zero-macrospin approach does not aim at reducing a single rotation to zero, but to reduce the overall rotations at the element level. This is achieved using a simple and direct approach (Izzuddin and Liang 2016) by considering the fact that rigid rotations can be removed if the geometric transformation from the co-rotational system to the deformed configuration is associated with only a stretch operation.

In applying the co-rotational approach to the proposed flat shell element, the co-rotational transformations are considered in two separate stages. In the first stage, the global element parameters are transformed to local parameters system, where only the basic nodal parameters are subject to transformation, with the additional hierarchic parameters coupled directly between adjacent elements (Izzuddin 2005). This then allows the local element response to be established, where in a second 
stage two additional transformations are performed to transform the local resistance forces and the local tangent stiffness matrix to the corresponding global entities. Similar to the first stage, no co-rotational transformation is required for entities associated with the additional hierarchic DOF, since these are edge based and are directly shared adjacent elements. It is worth noting though that, unlike the rectangular element developed by Izzuddin et al.(2004), local transformation of the hierarchic parameters is required from their edge-based system to the local element Cartesian system, though this transformation is constant throughout the geometric nonlinear analysis.

\section{Element Verification Tests}

To demonstrate the efficiency and convergence characteristics of the proposed element for geometric linear and analysis, several numerical examples are considered in this section. With regard to numerical integration, it is noted that all problems within this section utilise $7 \times 3$ Gauss points per element, corresponding to the number of Gauss points used for integration over the element planar domain and out of plane, respectively. It is also noted that for the presented problems, sufficient accuracy could have been achieved with fewer Gauss points, with 3 and 6 integration points sufficient for accurate integration of the basic linear and higher-order quadratic element forms.

\subsection{Square plate subjected to a point load}

This test considers a square plate clamped along two opposite edges and subject to a point load (P) applied at point (A) (Figure 5). Three different meshes, each with two different hierarchic forms, are considered for this problem. The first two meshes are based on a regular discretisation of the domain (Figure 5a-b), while the third one is based on a randomly generated triangulation of the domain (Figure 5c). Out of plane displacements at points (A) and (B) of each of these meshes in both their hierarchic linear (Figure 6a) and quadratic forms (Figure 6 b) are compared against the corresponding results from a similar numerical test performed by Izzuddin (2005). The list of abbreviations for the employed models is provided in Table 1.

The predicted displacements for point $\mathrm{A}$ and $\mathrm{B}$ at the final load step $(\mathrm{P}=250 \mathrm{kN})$, as well as relative errors compared to the solution of Izzuddin (2005) for all considered options are presented in Table 2 for linear shape functions and Table 3 for quadratic form.

The results show a good correlation for both linear and quadratic forms at all discretisation levels. As expected, the linear form of the triangular element provides a stiffer response compared to the rectangular element, but with mesh refinement the respective responses become more comparable (Table 2,3). The quadratic forms of both rectangular and triangular elements allow a better approximation even when using fewer elements. It is worth noting that the irregular mesh of triangular elements with quadratic terms shows a low level of relative error despite having a variety of elements with sub-optimal shapes. Some of 
this accuracy though is attributed to the fact that due to the random distribution within the domain, a higher density of nodes is obtained in the vicinity of points (A) and (B) compared to the CSL3 $10 \times 10$ case. Further analysis on the effects of mesh irregularity indicated that mesh variations can result in an average error of $2.35 \%$ and $1.52 \%$ for the deflections at points A and $\mathrm{B}$, respectively.

This test is also used to assess the computational efficiency of the developed element. To this end, the time required for the analysis as well as the prediction accuracy are compared against models utilising a 4-noded rectangular shell element (CSL4) (Izzuddin, Tao and Elghazouli, 2004), a 9-noded quadrilateral element (CVS9 - H3O9) (Izzuddin and Liang, 2017) and a 6-noded triangular element (CVS6 - H3O6) (Liang, 2015). The developed element and CSL4 elements are considered in both quadratic (Q) and linear (L) hierarchic forms. In order to achieve comparable accuracy, similar meshes based on a $20 \times 20$ configuration are used for the CSL3, CSL4 and CVS6 models, while a $10 \times 10$ mesh is used for the CVS9 model. The normalised errors and computing times are resented in Figure 7.

The obtained results demonstrate the higher efficiency of the quadratic hierarchic form of the developed traingular element (CSL3-Q) compared to the conventional quadratic element (CVS6) of the same mesh density. It should also be noted that while the linear hierarchic form (CSL3-L) is less accurate than the quadratic form (CSL3-Q), it offers a significant reduction in computing time without a significant negative impact on accuracy. As expected, due to the difference in the number of elements required for the triangular and rectangular meshes of the same density, the latter marginally outperforms the former in term of efficiency for similar levels of accuracy. However, a key benefit of the triangular element is its applicability to the modelling of irregular structural forms.

\subsection{Buckling of rectangular plate under planar loading}

This test considers a rectangular plate simply supported along four edges under a uniformly distributed load $(p)$ applied along the plate short sides (Figure 8). To ensure that the system follows the post-buckling path associate with the lowest buckling mode, a small out of plane load $\left(10^{-2} \mathrm{~N}\right)$ is applied at point A. Due to symmetry only quarter of the plate is modelled. As in the previous test, two different mesh densities $(15 \times 5 \times 2 ; 30 \times 10 \times 2)$ and two element forms (linear and quadratic) are considered for each test (Figure 9).

The obtained out-of-plane displacement at point A and the relative errors compared to the solution of Izzuddin (2005) are presented for all considered options in Table 4. The results obtained with a fine mesh of quadratic rectangular elements at the end of analysis are used as a basis for error estimation and are highlighted in the tables. 
It is noted that all models converge with mesh refinement, where the performance of the quadratic forms of both triangular and quadrilateral elements is generally better, as would be expected. It is also important to underline that this test involves a buckling mode with multiple waves over the plate, which requires a relatively fine mesh for an accurate representation.

\section{Element Validation against Experimental Results}

This section addresses the applicability of the proposed triangular shell element to the assessment of reinforced concrete slabs under extreme loading, where two experimental tests are considered for validation purposes.

\subsection{Octagonal two-way concrete slab subjected to point load}

The first test relates to the experimental work conducted by Fall et al. (2014). The experiment aimed at investigating the applicability of yield line assessment for a two-way slab made of fibre-reinforced concrete. The current work only makes use of the results from the first control specimen presented in the aforementioned study, which relates to a concrete slab with only rebar reinforcement. To reduce the influence of complex boundary conditions on the system response, Fall et al. (2014) considered an octagonal slab simply supported and laterally unrestrained on 4 opposite sides (Figure 10). The specimen was reinforced with a single layer of $\varnothing 6 \mathrm{~mm}$ rebars, where the influence of the load redistribution mechanism was studied using unequal rebar spacing in the two directions, specifically $194 \mathrm{~mm}$ and $96 \mathrm{~mm}$, as illustrated in Figure 10.

For the current numerical model, the material parameters are taken from the original paper and presented in Table 5. A fixed-crack elevated-temperature model developed by Izzuddin et al. (2004) is used to describe the concrete behaviour. This model is capable of dealing with both the nonlinear compressive and tensile response of concrete. Compressive nonlinearity in the biaxial response of concrete is included via an evolving plastic interaction surface, while the tensile response is modelled by means of separate strength envelopes for the biaxial stresses. On the other hand, a standard uniaxial elasto-plastic model with kinematic strain-hardening is assumed for the reinforcement material, where an elliptical post-yield response is considered up to the ultimate strength (Song et al., 2000). Good accuracy of numerical integration was achieved for this problem using $7 \times 5$ Gauss points per element.

The results of the developed numerical model demonstrate good agreement with the experimental results of Fall et al. (2014), as shown in Figure 11 for the centre point deflection. It can be seen that proposed element is capable of capturing with sufficient accuracy both the first peak load and the subsequent nonlinear large displacement response dominated by membrane action. It is worth noting that convergence difficulties were encountered beyond the ultimate load level due to extensive damage of concrete material with the rebars simultaneously attaining the ultimate strength. The developed numerical model is also 
capable of predicting the cracked regions with sufficient accuracy, where the corresponding crack patterns can be seen in Figure 11. Some of the deviations in the response can be attributed to the stochastic nature of the experimental concrete response and the deterministic nature of the computer-based simulation.

\subsection{Circular concrete plate subjected to uniformly distributed load}

The second test relates to the experiments conducted by Chen et al. (1990), where an approach for miniaturised testing was developed. To validate the proposed approach, Chen et al. (1990) performed a series of tests on miniaturised circular slabs. Consideration is given here to one of the specimens from the experimental programme.

The considered specimen had a continuous support along the circumference of the slab, which is modelled by restraining all the nodes on the circumference and ensuring that edges have zero displacement by enforcing zero values for parameters of related hierarchic DOF. The specimen was also doubly reinforced with uniformly spaced rectangular wire mesh (Figure 12). This reinforcement was effectively modelled with the previously described algorithm for defining rebar orientation relative to the local element system.

The material properties for this test are taken from the data provided by Chen et al. (1990). The same material models are used as in the previous test, with the associated material properties given in Table 5.

It is evident from the results in Figure 13 that the proposed element is capable of predicting the nonlinear response of the circular slab, including the influence of tensile cracking and yielding of the reinforcement. It is unclear whether the small discrepancy in modelling the post-yield response is due to inaccurate material properties or reporting of the experimental results, though the numerical predictions are generally good considering the small scale of the specimen and material uncertainty. Interestingly in the post-yield range, a compression ring (Figure 13) is formed after significant displacement, which serves as a well-known restraint for membrane action within the slab, where similar effects of relatively early onset of hardening for circular slabs have been previously reported (Herraiz and Vogel, 2016).

\section{Large-Scale Application of Shell Element}

To demonstrate the applicability of the developed element, it is employed here in the nonlinear analysis of a large irregular 44 storey structure subjected to a sudden column loss, similar in form to the Gherkin building in London (Massey, 2014). The irregularity of the considered building relates to both its diagrid structural system (Figure 14) and to the floor plan that changes along the structure vertical axis (Figure 15). Columns, girders and diagrid system are modelled with beam elements 
(Izzuddin, 2001). Different parts of the slab are assigned with different rebar layout, utilising the capability of developed procedure to assign rebar orientation for each element relatively easily.

Due to the large overall problem size of 213673 nodes and 219573 elements, a recently developed partitioned modelling approach (Jokhio and Izzuddin, 2015) is employed to take advantage of parallel computing capabilities, where 44 partitions are used. The most efficient approach to partitioning the analysed structure consists of locating the partition boundaries between the individual floors, as this minimises the nodes on the partitioned boundary and maximises interior nodes, thus enabling significant efficiencies in parallel processing of the partitioned model (Jokhio and Izzuddin 2015).

To assess the influence of slab elements on the overall structural response to the localised damage, a series of analyses with and without the developed elements have been performed. Each analysis considered a specific damage scenario such as removal of internal column, removal of two neighbouring internal columns, removal of a diagrid pair and combinations of the above. These damage conditions were considered also at different elevations, specifically at the first, sixth and twelfth floor levels. The undertaken analysis highlighted the robustness of the considered diagrid structural system, which agrees with previous assessments conducted by other researchers (Kim and Lee, 2010; Kim and Hong, 2011; Kim and Jung, 2013; Kwon and Kim, 2014). However, it is noted that previous works disregarded the influence of the floor slabs, either due to computational demand or due to the absence of accurate nonlinear elements for such structural systems. Our findings, on the other hand, indicate that such an assessment can lead to overly conservative results for some of the possible damage scenarios. In all of the analysis undertaken for the present work, it can be observed that slabs in the affected bays are subject to significant deformation, as illustrated in Figure 16 (for the case of removal of two neighbouring internal columns), which leads to considerable membrane action, as also reported in a recent experimental study conducted by Dat and Hai (2013). It should also be noted that disregarding membrane action will only be conservative if the floor system is capable of undergoing large deflections without losing structural integrity, otherwise as reported by Vlassis et al. (2009) the impact of the collapsing floor is very likely to trigger progressive collapse of the considered structure. As such it is evident that for a realistic assessment of structural robustness, a detailed slab model has to be considered, which is demonstrably provided by the developed triangular element.

\section{Conclusions}

This paper introduces a novel triangular shell element for the nonlinear analysis of RC floor slabs subjected to extreme loading conditions. The developed shell element is formulated in a local reference system based on the conventional ReissnerMindlin hypothesis. The element is introduced in two hierarchic forms, linear and quadratic, the latter allowing for quadratic 
approximation of displacement fields at any point of the considered slab. The paper presents the details of the local element formulation, as well as its incorporation within a co-rotational framework for large displacement analysis.

The new shell element has been implemented within the nonlinear structural analysis program $A D A P T I C$, which is employed in this paper to provide verification and validation of the element formulation and its implementation. Comparisons against existing rectangular shell element demonstrate very good agreement in both the small and large displacement ranges for several test problems. The applicability of the element to the modelling of irregular slabs is first validated via comparisons against experimental results of reinforced concrete slabs of different shapes and reinforcements ratios. The agreement between numerical and experimental data demonstrate a very good overall accuracy in predicting both the deflections and load capacity. Finally, the nonlinear analysis of a large scale irregular structure subject to sudden loss of one or more structural elements is presented. The results of this study confirm the need for detailed modelling of concrete slabs to adequately assess structural robustness.

Finally, the development of the proposed element paves the way for a wide range of applications on large scale and irregular building structures subject to extreme loading conditions, particularly where full high fidelity models are required, such as under earthquake, global blast and structural robustness scenarios.

\section{Acknowledgment}

The authors acknowledge the Research Computing Service at Imperial College for providing and supporting the required High Performance Computing facilities.

\section{References}

Belytschko, T., Liu, W. K. and Moran, B. (2000) Nonlinear Finite Elements For Continua And Structures. Chichester: John Wiley \& Sons, Inc.

Calavera, J. (2012) 'Manual for Detailing Reinforced Concrete Structures to EC2'. London: CRC Press.

Chen, Y. J., Chen, H. L., Dancygier, A. N., Shah, S. P. and Keer, L. M. (1990) 'Tests of model reinforced concrete circular slabs', ACI Structural Journal, 87(6), pp. 727-737.

Crisfield, M. A. (2000a) Non-Linear Finite Element Analysis of Solids and Structures - Volume 1: Essentials. Chichester: John Wiley \& Sons, Inc.

Crisfield, M. A. (2000b) Non-Linear Finite Element Analysis of Solids and Structures - Volume 2: Advanced Topics. 2nd edn. Chichester: John Wiley \& Sons, Inc.

Dat, P. X. and Hai, T. K. (2013) 'Membrane actions of RC slabs in mitigating progressive collapse of building 
structures', Engineering Structures. Elsevier Ltd, 55, pp. 107-115. doi: 10.1016/j.engstruct.2011.08.039.

Dunavant, D. A. (1985) 'High degree efficient symmetrical Gaussian quadrature rules for the triangle', International Journal for Numerical Methods in Engineering, 21(6), pp. 1129-1148. doi: 10.1002/nme.1620210612.

Dvorkin, E. N. and Bathe, K. (1984) 'A continuum mechanics based four-node shell element for general non-linear analysis', Engineering Computations, 1(1), pp. 77-88. doi: 10.1108/eb023562.

Fall, D., Shu, J., Rempling, R., Lundgren, K. and Zandi, K. (2014) 'Two-way slabs: Experimental investigation of load redistributions in steel fibre reinforced concrete', Engineering Structures, 80, pp. 61-74. doi: 10.1016/j.engstruct.2014.08.033.

Herraiz, B. and Vogel, T. (2016) 'Novel design approach for the analysis of laterally unrestrained reinforced concrete slabs considering membrane action', Engineering Structures. Elsevier Ltd, 123, pp. 313-329. doi: 10.1016/j.engstruct.2016.05.033.

Hughes, T. J. R. and Tezduyar, T. E. (1981) 'Finite Elements Based Upon Mindlin Plate Theory With Particular Reference to the Four-Node Bilinear Isoparametric Element', Journal of Applied Mechanics, 48(3), p. 587. doi: 10.1115/1.3157679.

Izzuddin, B. A. (1991) Nonlinear Dynamic Analysis of Framed Structures. Ph.D. Thesis, Imperial College, University of London, London. Available at: spiral:8443/handle/10044/1/8080.

Izzuddin, B. A. (2001) 'Conceptual issues in geometrically nonlinear analysis of 3D framed structures', Computer Methods in Applied Mechanics and Engineering, 191(8-10), pp. 1029-1053. doi: 10.1016/S0045-7825(01)00317-6.

Izzuddin, B. A. (2005) 'An enhanced co-rotational approach for large displacement analysis of plates', International Journal for Numerical Methods in Engineering, 64(10), pp. 1350-1374. doi: 10.1002/nme.1415.

Izzuddin, B. A. and Liang, Y. (2016) 'Bisector and zero-macrospin co-rotational systems for shell elements', International Journal for Numerical Methods in Engineering, 105(4), pp. 286-320. doi: 10.1002/nme.4978.

Izzuddin, B. A. and Liang, Y. (2017) 'A hierarchic optimisation approach towards locking-free shell finite elements', Computers and Structures. Elsevier Ltd. doi: 10.1016/j.compstruc.2017.08.010.

Izzuddin, B. A., Tao, X. Y. and Elghazouli, A. Y. (2004) 'Realistic Modeling of Composite and Reinforced Concrete Floor Slabs under Extreme Loading. I: Analytical Method', Journal of Structural Engineering, 130(12), pp. 1972-1984. doi: 10.1061/(ASCE)0733-9445(2004)130:12(1972).

Jokhio, G. A. and Izzuddin, B. A. (2015) 'A Dual Super-Element Domain Decomposition Approach for Parallel Nonlinear Finite Element Analysis', International Journal for Computational Methods in Engineering Science and Mechanics, 16(3), pp. 188-212. doi: 10.1080/15502287.2015.1043163.

Kim, J. and Hong, S. (2011) 'Progressive collapse performance of irregular buildings', The Structural Design of Tall and Special Buildings, 20(1), pp. 721-734. doi: 10.1002/tal.

Kim, J. and Jung, M.-K. (2013) 'Progressive collapse resisting capacity of tilted building structures', The Structural 
Design of Tall and Special Buildings, 22(18), pp. 1359-1375. doi: 10.1002/tal.1010.

Kim, J. and Lee, Y.-H. (2010) 'Progressive collapse resisting capacity of tube-type structures', The Structural Design of Tall and Special Buildings, 19, pp. 761-777. doi: 10.1002/tal.512.

Ko, Y., Lee, P. S. and Bathe, K. J. (2017) 'A new MITC4+ shell element', Computers and Structures. Elsevier Ltd, 182, pp. 404-418. doi: 10.1016/j.compstruc.2016.11.004.

Kwon, K. and Kim, J. (2014) 'Progressive Collapse and Seismic Performance of Twisted Diagrid Buildings', International Journal of High-Rise Buildings, 3(3), pp. 223-230.

Lee, Y., Lee, P. S. and Bathe, K. J. (2014) 'The MITC3+ shell element and its performance', Computers and Structures. Elsevier Ltd, 138, pp. 12-23. doi: 10.1016/j.compstruc.2014.02.005.

Liang, Y. (2015) Nonlinear Analysis of Composite Shells with Application to Glass Structures. PhD thesis, Imperial College London Department.

Liu, Y. and Teng, S. (2008) 'Nonlinear Analysis of Reinforced Concrete Slabs Using Nonlayered Shell Element', Journal of Structural Engineering, 134(7), pp. 1092-1100. doi: 10.1061/(ASCE)0733-9445(2008)134:7(1092).

Massey, J. (2014) 'Risk Design', Grey Room, 54, pp. 6-33. doi: 10.1162/GREY_a_00134.

Schofield, J. (2012) 'Case study: Capital Gate', CTBUH Journal. International Journal on Tall Buildings and Urban Habitat, (8), pp. 12-17. Available at: http://technicalpapers.ctbuh.org.

Shin, M., Kang, T. H.-K. and Grossman, J. S. (2009) 'Practical modelling of high-rise dual systems with reinforced concrete slab-column frames', The Structural Design of Tall and Special Buildings, 749(March 2009), pp. 728-749. doi: 10.1002/tal.509.

Song, H.-W., Shim, S.-H., Byun, K.-J. and Maekawa, K. (2002) 'Failure analysis of reinforced concrete shell structures using layered shell element with pressure node', Journal of Structural Engineering, 128(5), pp. 655-664. doi: 10.1061/(ASCE)0733-9445(2002)128:5(655).

Song, L., Izzuddin, B. A., Elnashai, A. S. and Dowling, P. J. (2000) 'An integrated adaptive environment for fire and explosion analysis of steel frames — Part I':, Journal of Constructional Steel Research, 53(1), pp. 63-85. doi: 10.1016/S0143974X(99)00040-1.

Swamy Naidu, N. V. and Sateesh, B. (2015) 'Improved Bilinear Degenerated Shell Element', International Journal of Computational Methods, 12(02), p. 1550004. doi: 10.1142/S0219876215500048.

Vlassis, A. G., Izzuddin, B. A., Elghazouli, A. Y. and Nethercot, D. A. (2009) 'Progressive collapse of multi-storey buildings due to failed floor impact', Engineering Structures, 31(7), pp. 1522-1534. doi: 10.1016/j.engstruct.2009.02.009.

Zhang, Y. X. and Zhu, Y. (2010) 'A new shear-flexible FRP-reinforced concrete slab element', Composite Structures. Elsevier Ltd, 92(3), pp. 730-735. doi: 10.1016/j.compstruct.2009.09.013.

Zienkiewicz, O. C. and Taylor, R. L. (2000a) The Finite Element Method Volume 1: The Basis. 5th edn. Oxford: 


\section{Butterworth-Heinemann.}

Zienkiewicz, O. C. and Taylor, R. L. (2000b) The Finite Element Method Volume 2: Solid Mechanics. 5th edn. Butterworth-Heineman. 


\section{Table captions}

Table 1. Definition of abbreviations for considered tests

Table 2. Deflection and relative errors for linear form shape-function meshes

Table 3. Deflection and relative errors for quadratic form shape-function meshes

Table 4. Displacement and relative errors for considered options

Table 5. Material properties employed in numerical analysis for octagonal and circular slabs 


\section{Figure captions}

Figure 1.Element geometry and coordinate systems:

a) Local coordinate system; b) Natural area coordinate system; c) Rebar orientation diagram

Figure 2. Orthogonal vector rebar orientation approach

Figure 3. Physical meaning of hierarchic shape functions

Figure 4. Alternative local reference systems: a) bisector definition, and b) zero-macrospin definition

Figure 5. Square plate problem setup and employed mesh

a) regular $10 \times 10 \times 2$ elements; b) regular $20 \times 20 \times 2$ elements; c) irregular 209 elements

Figure 6. Results comparison for considered mesh options:

1) coarse mesh, 2) fine mesh, 3) irregular mesh,

a) linear form, b) quadratic form

Figure 7. Computational efficiency comparison

Figure 8. Buckling problem set-up with two employed mesh densities overlay

Figure 9. Comparison of lateral displacement at point A for different mesh densities and hierarchic forms

Figure 10. Octagonal slab two way bending test setup and employed mesh (dimensions in mm)

Figure 11. Displacement of the octagonal slab centre, and experimental/numerical crack pattern

Figure 12. Circular slab experimental setup and employed finite element model

Figure 13. Centre point deflections of circular concrete slab; inset contours refer to principal concrete stress (MPa) over the bottom surface

Figure 14. 3-D view of analysed structure

Figure 2. Irregular structure meshed floor plan

Figure 3. Slab deflections for the affected bay for the case of removal of two neighbouring internal columns 

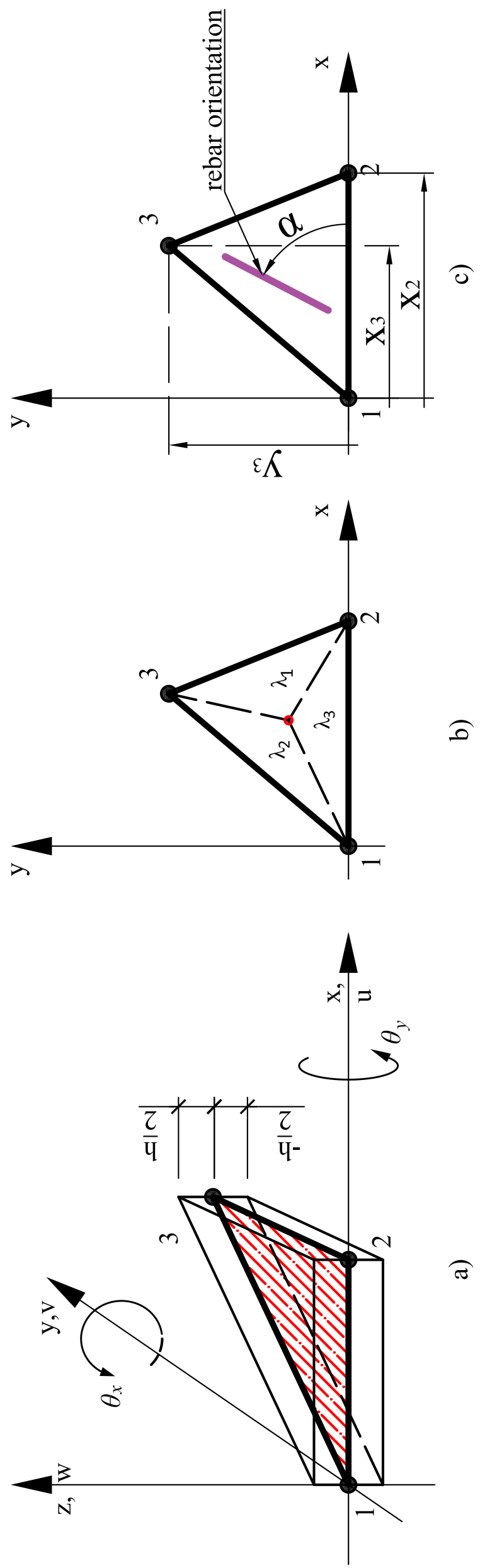


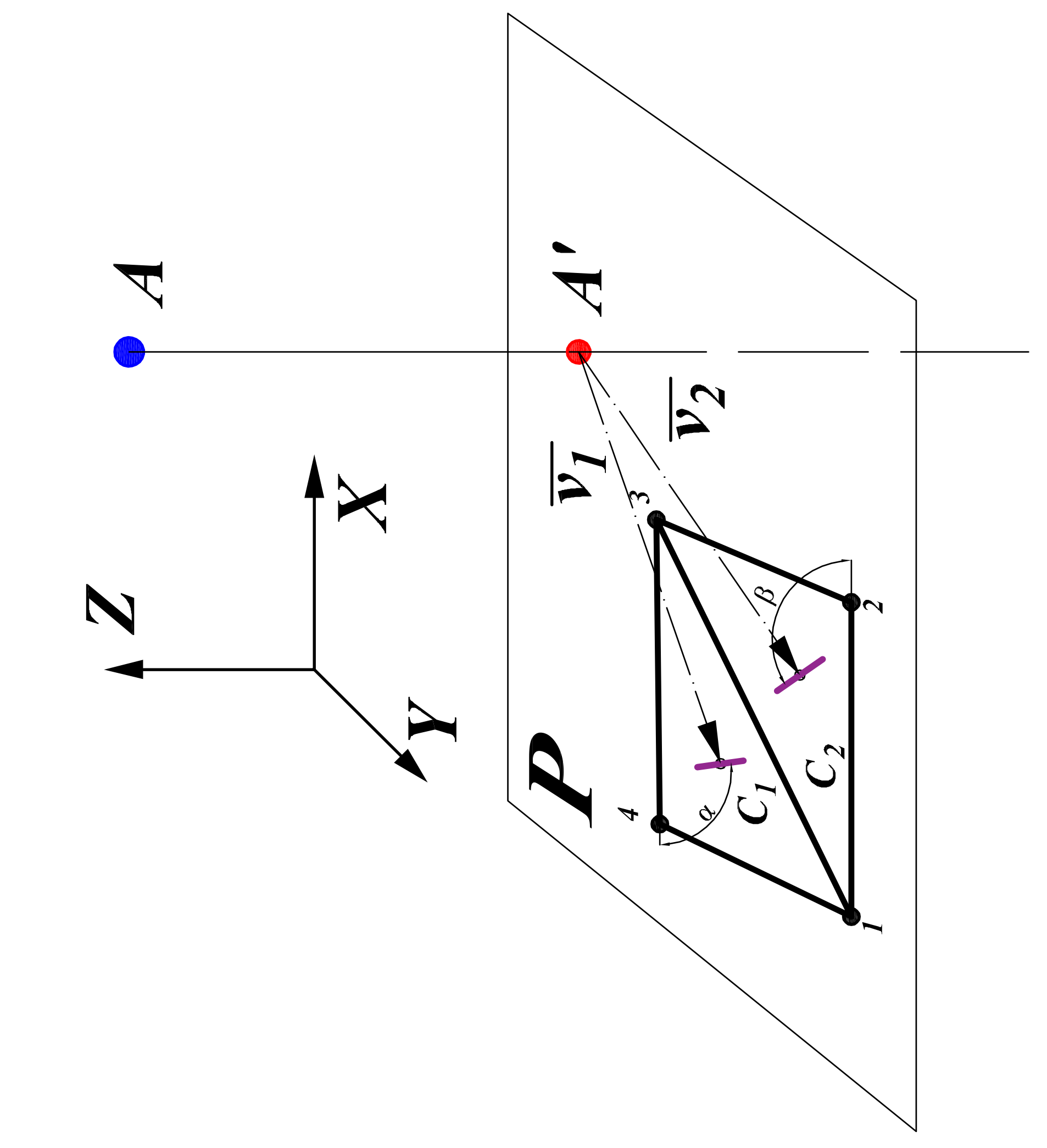

Figure 2. Orthogonal vector rebar orientation approach 

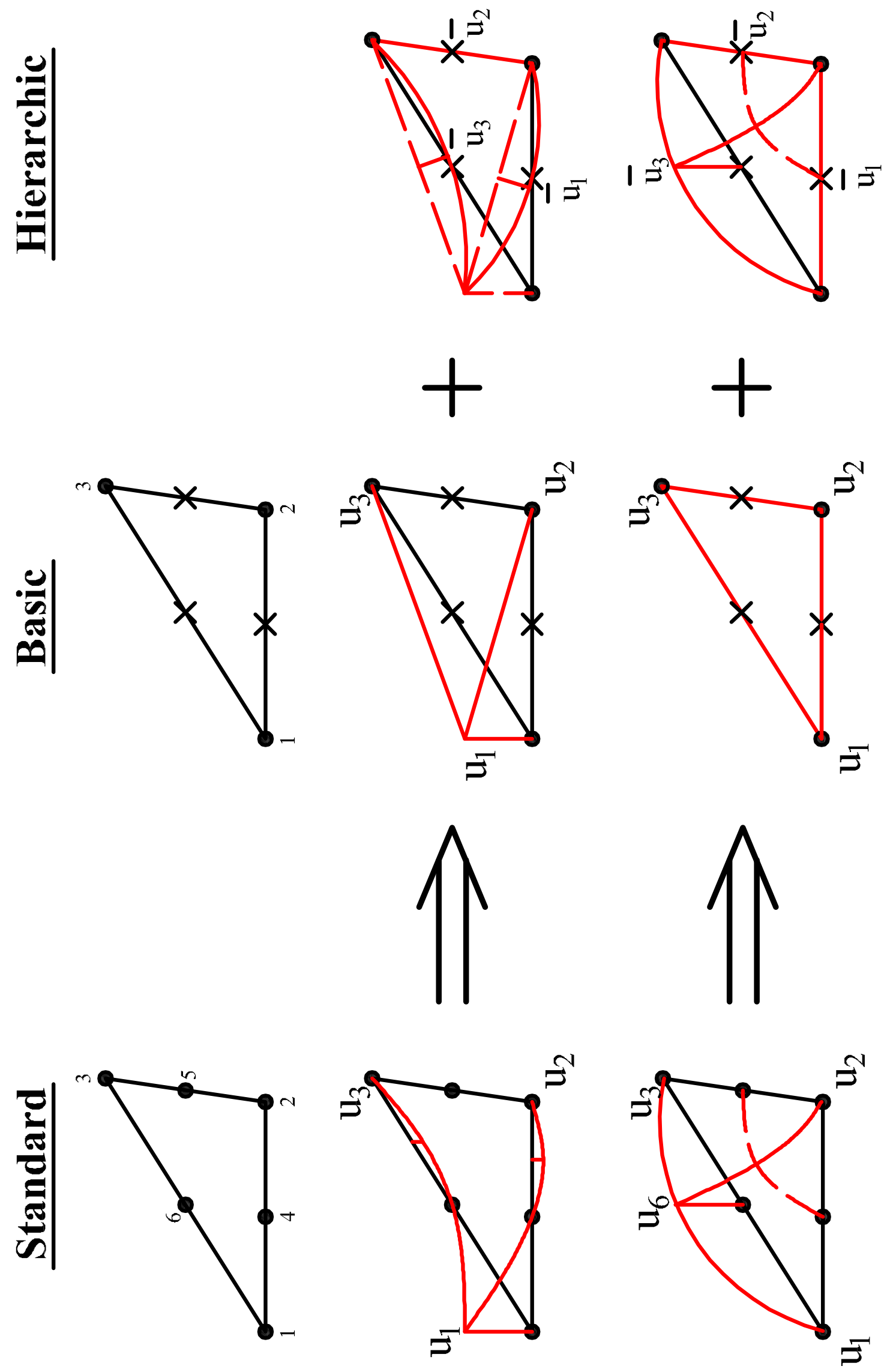
Figure 4. Alternative local reference systems: a) bisector definition, and b) zero-macrospin definition
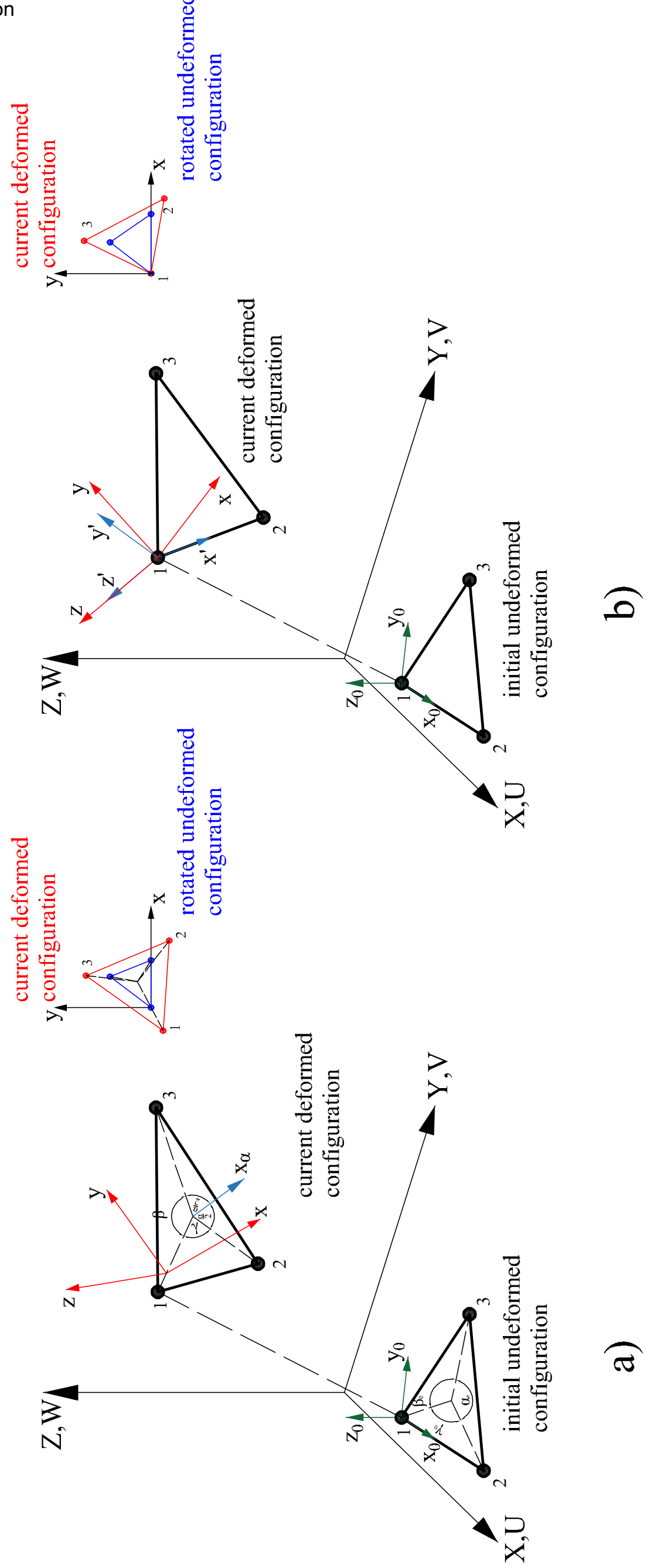

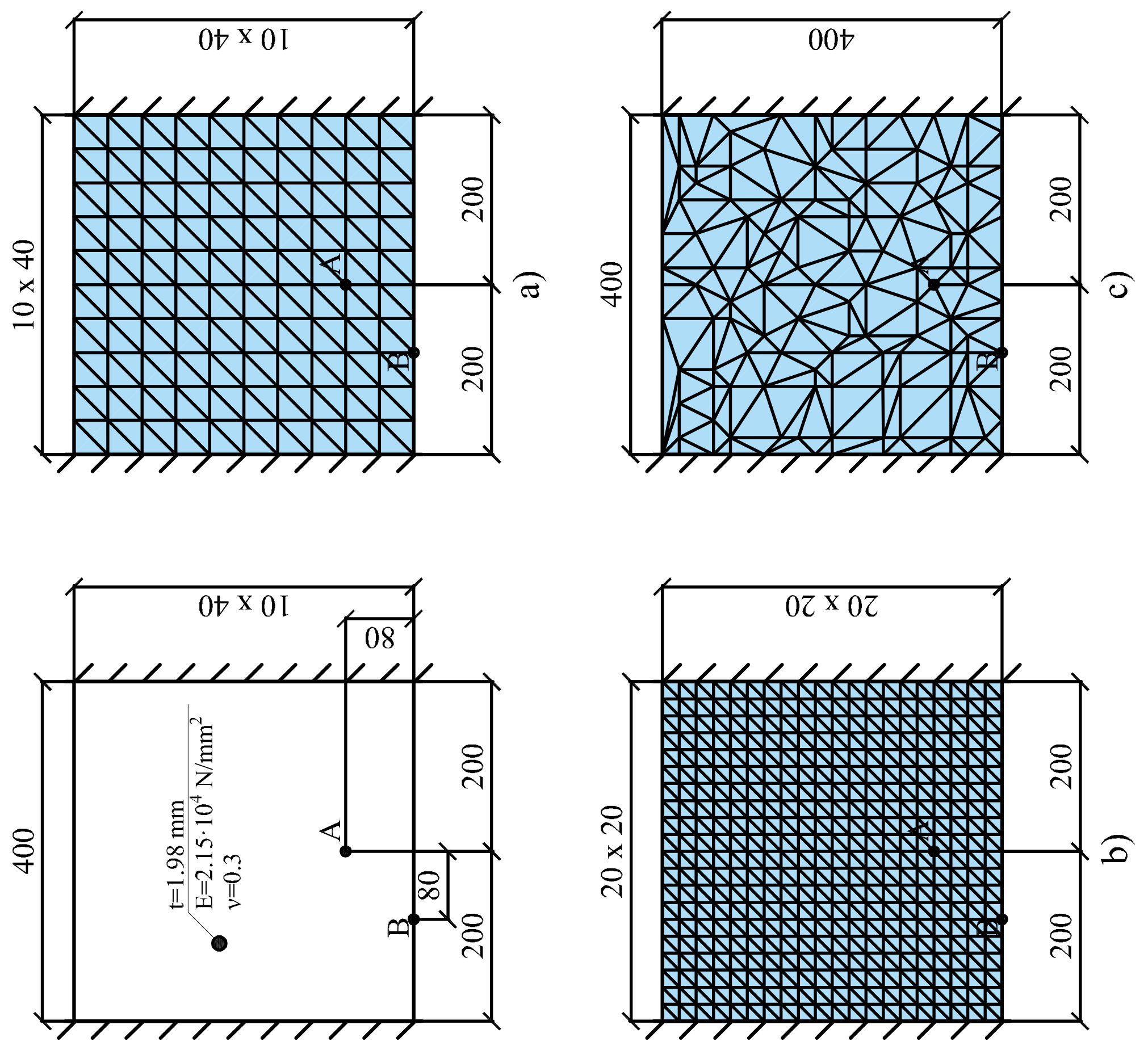


\begin{tabular}{|c|l|}
\hline Abbreviation & \multicolumn{1}{|c|}{ Definition } \\
\hline $\mathbf{C S L 3 - Q ( L )}$ & Mesh of $\mathrm{M} \times \mathrm{N}$ 3-noded elements (each rectangle divided into 2 triangles). \\
$\mathbf{M} \times \mathbf{N} \times \mathbf{2}$ & L: linear variant, Q: quadratic variant. \\
\hline $\begin{array}{c}\mathbf{C S L 3 - Q}(\mathbf{L}) \\
\mathbf{2 0 9}\end{array}$ & Irregular mesh consisting of 209 triangular elements. \\
\hline $\begin{array}{c}\mathbf{C S L 4 - Q ( L )} \\
\mathbf{M} \times \mathbf{N}\end{array}$ & Mesh of of $\mathrm{M} \times \mathrm{N}$ 4-noded rectangular elements. \\
\hline
\end{tabular}


Figure 6. Results comparison for considered mesh options: 1 ) coarse mesh, 2) fine mesh, 3) irregular mesh, a) linear form, b)
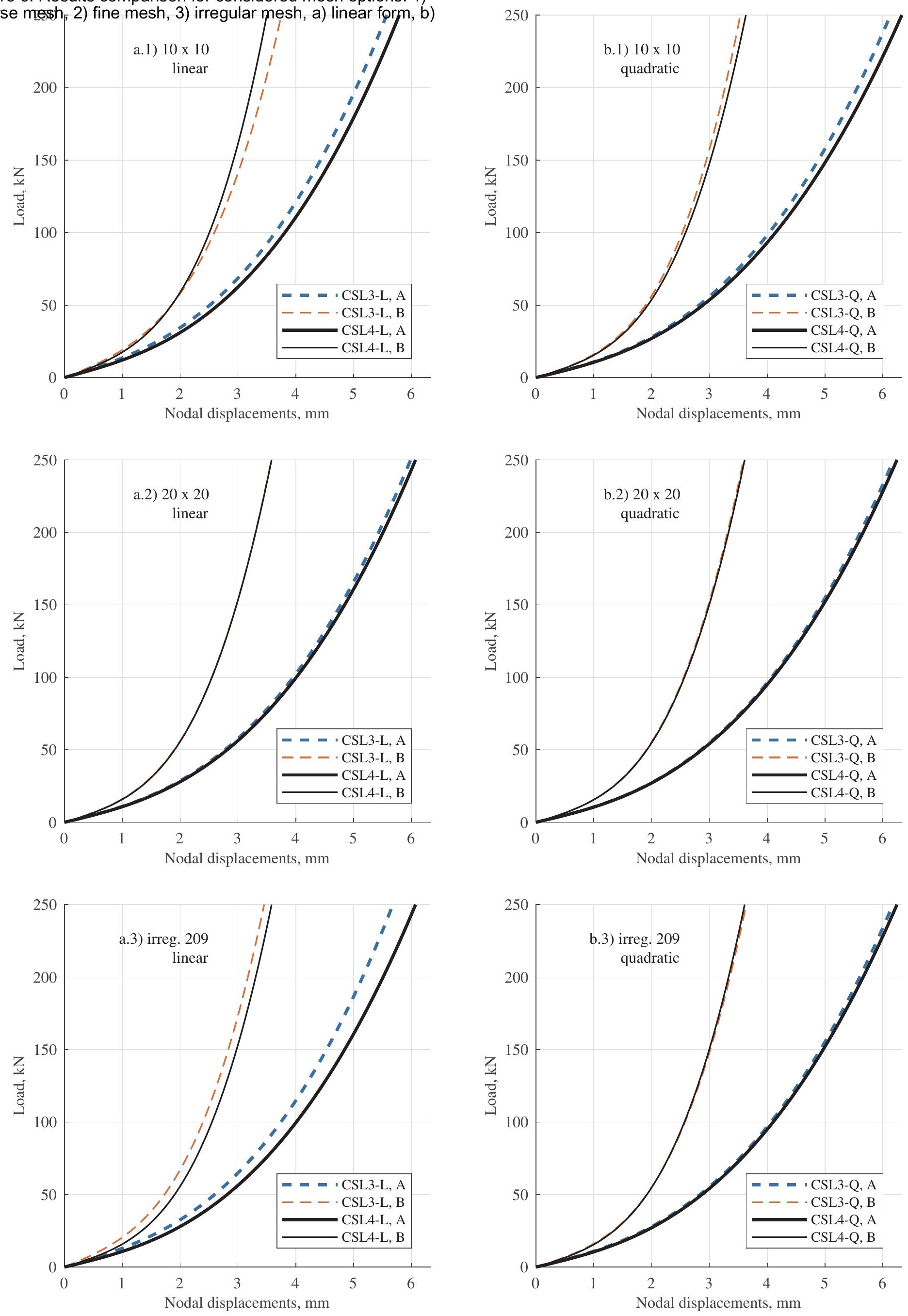
Table 2. Deflection and relative errors for linear form shapefunction meshes

Click here to download Table table2.xlsx $\doteq$

\begin{tabular}{|c|c|c|c|c|c|c|c|c|}
\hline \multirow[t]{2}{*}{ Points } & \multirow{2}{*}{$\begin{array}{c}\text { CSL4-L } \\
\mathbf{1 0 \times 1 0} \\
\text { Disp. mm }\end{array}$} & \multicolumn{2}{|c|}{$\begin{array}{c}\text { CSL3-L } \\
10 \times 10 \times 2\end{array}$} & \multirow{2}{*}{$\begin{array}{c}\text { CSL4-L } \\
\mathbf{2 0 \times \mathbf { 2 0 }} \\
\text { Disp. mm }\end{array}$} & \multicolumn{2}{|c|}{$\begin{array}{c}\text { CSL3-L } \\
20 \times 20 \times 2\end{array}$} & \multicolumn{2}{|c|}{$\begin{array}{c}\text { CSL3-L } \\
209\end{array}$} \\
\hline & & Disp. mm & Error \% & & Disp. mm & Error \% & Disp. mm & Error \% \\
\hline $\mathrm{A}$ & 5.7901 & 5.5711 & 3.78 & 6.0719 & 5.9863 & 1.41 & 5.6802 & 6.45 \\
\hline B & 3.4992 & 3.7513 & 7.21 & 3.5821 & 3.5786 & 0.1 & 3.4533 & 3.6 \\
\hline
\end{tabular}


Table 3. Deflection and relative errors for quadratic form shapefunction meshes

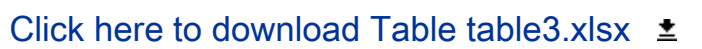

\begin{tabular}{|c|c|c|c|c|c|c|c|c|}
\hline \multirow{3}{*}{ Points } & CSL4-Q & \multicolumn{2}{|c|}{$\begin{array}{c}\text { CSL3-Q } \\
\mathbf{1 0 \times 1 0}\end{array}$} & \multicolumn{2}{|c|}{$\begin{array}{c}\text { CSL4-Q } \\
\text { 10 }\end{array}$} & \multicolumn{2}{c|}{$\begin{array}{c}\text { CSL3-Q } \\
\mathbf{2 0} \times \mathbf{2 0}\end{array}$} & \multicolumn{2}{c|}{$\begin{array}{c}\text { CSL3-Q } \\
\text { 20 } \times \mathbf{2}\end{array}$} & \multicolumn{2}{|c|}{$\mathbf{2 0 9}$} \\
\cline { 2 - 9 } & Disp. $\mathrm{mm}$ & Disp. mm & Error \% & Disp. $\mathrm{mm}$ & Disp. mm & Error \% & Disp. mm & Error \% \\
\hline A & 6.3277 & 6.1306 & 3.12 & 6.2433 & 6.186 & 0.92 & 6.1734 & 1.12 \\
\hline B & 3.6297 & 3.5341 & 2.63 & 3.6087 & 3.5889 & 0.55 & 3.6359 & 0.75 \\
\hline
\end{tabular}




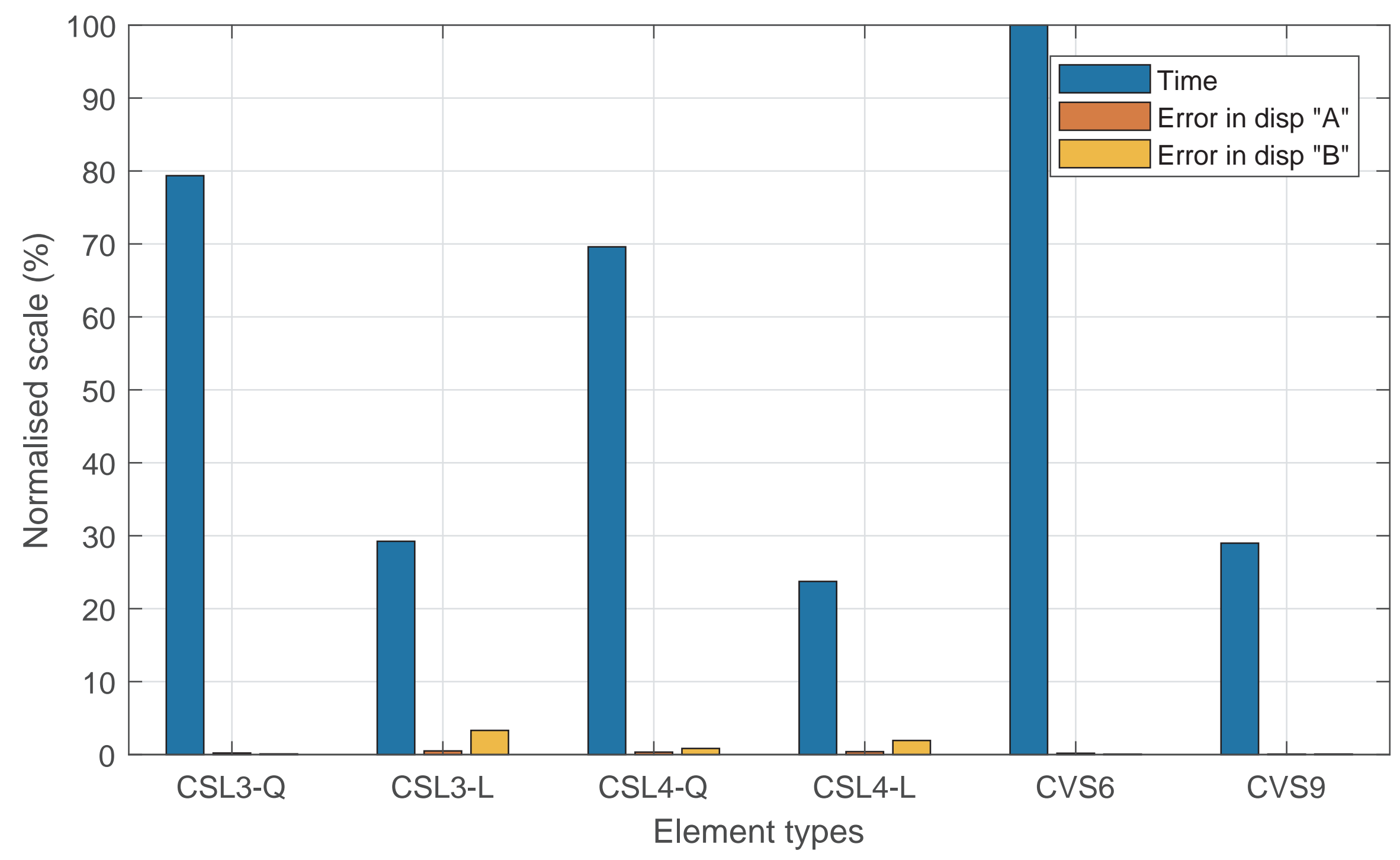




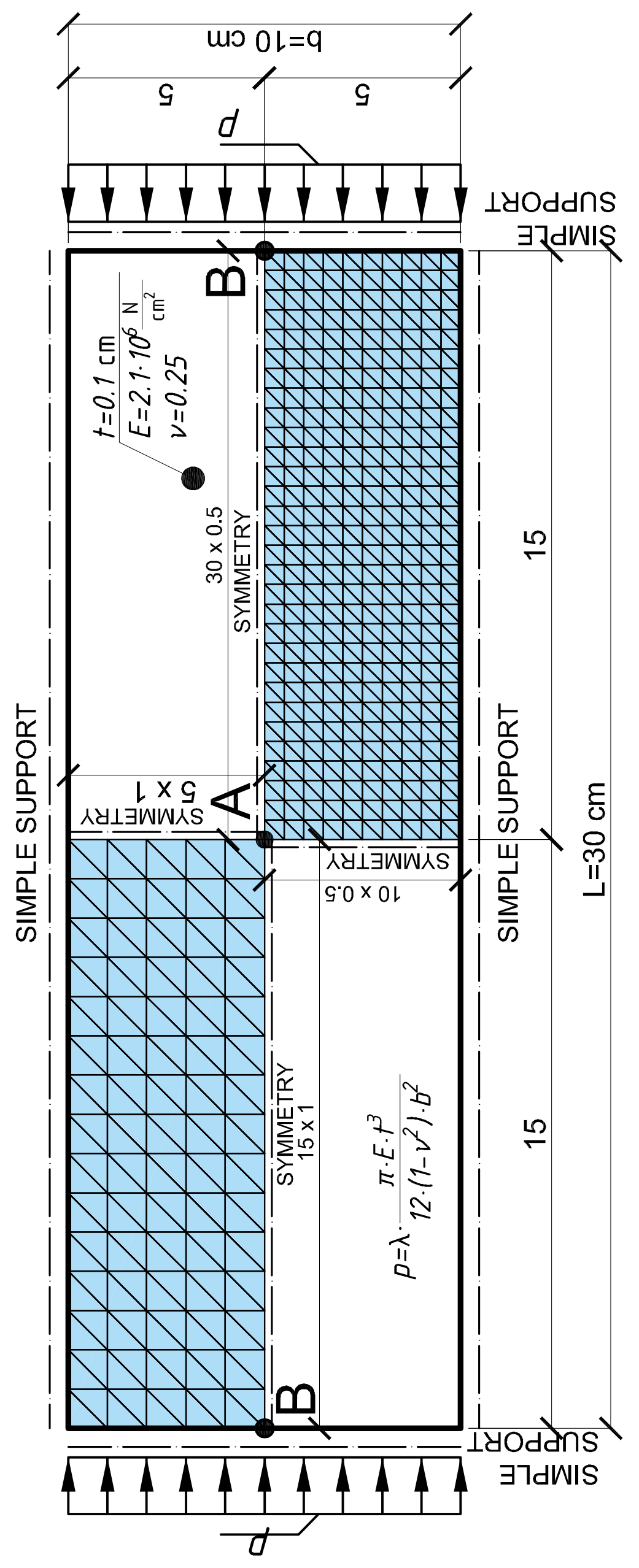




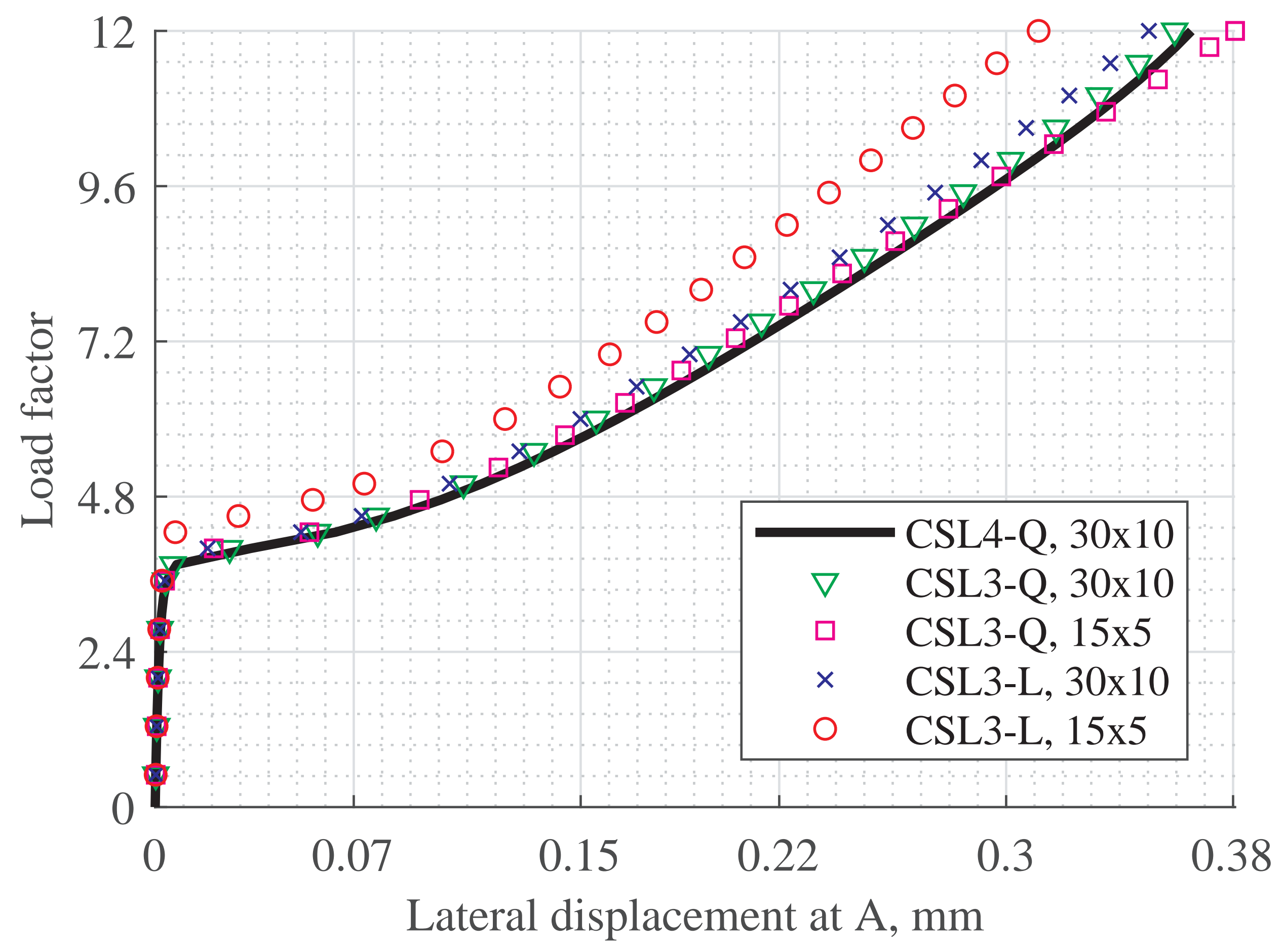




\begin{tabular}{|c|c|c|c|c|c|}
\hline \multirow{2}{*}{$\begin{array}{c}\text { Hierarchic } \\
\text { form }\end{array}$} & $\begin{array}{c}\text { CSL4 } \\
\mathbf{3 0 \times 1 0}\end{array}$ & \multicolumn{2}{|c|}{ CSL3 } & \multicolumn{2}{c|}{ CSL3 } \\
\cline { 2 - 6 } & $\begin{array}{c}\text { Disp., } \\
\mathrm{mm}\end{array}$ & $\begin{array}{c}\text { Disp., } \\
\mathrm{mm}\end{array}$ & $\begin{array}{c}\text { Error, } \\
\%\end{array}$ & $\begin{array}{c}\text { Disp., } \\
\mathrm{mm}\end{array}$ & $\begin{array}{c}\text { Error, } \\
\%\end{array}$ \\
\hline Linear & \multirow{2}{*}{0.365} & 0.311 & 14.795 & 0.35 & 4.110 \\
\cline { 3 - 6 } & 0.381 & 4.384 & 0.359 & 1.644 \\
\hline
\end{tabular}


Figure 10. Octagonal slab two way bending test setup and

employed mesh (dimensions in $\mathrm{mm}$ )
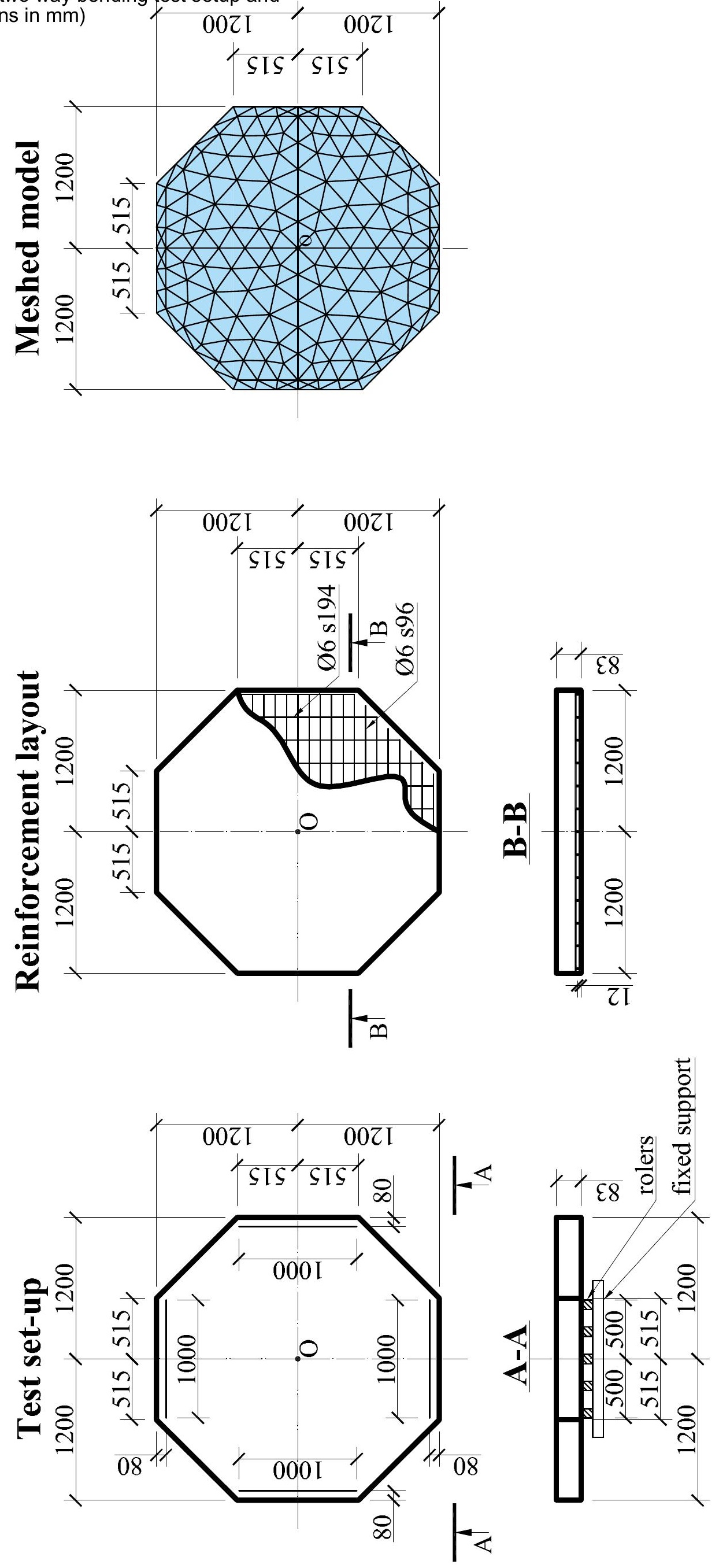


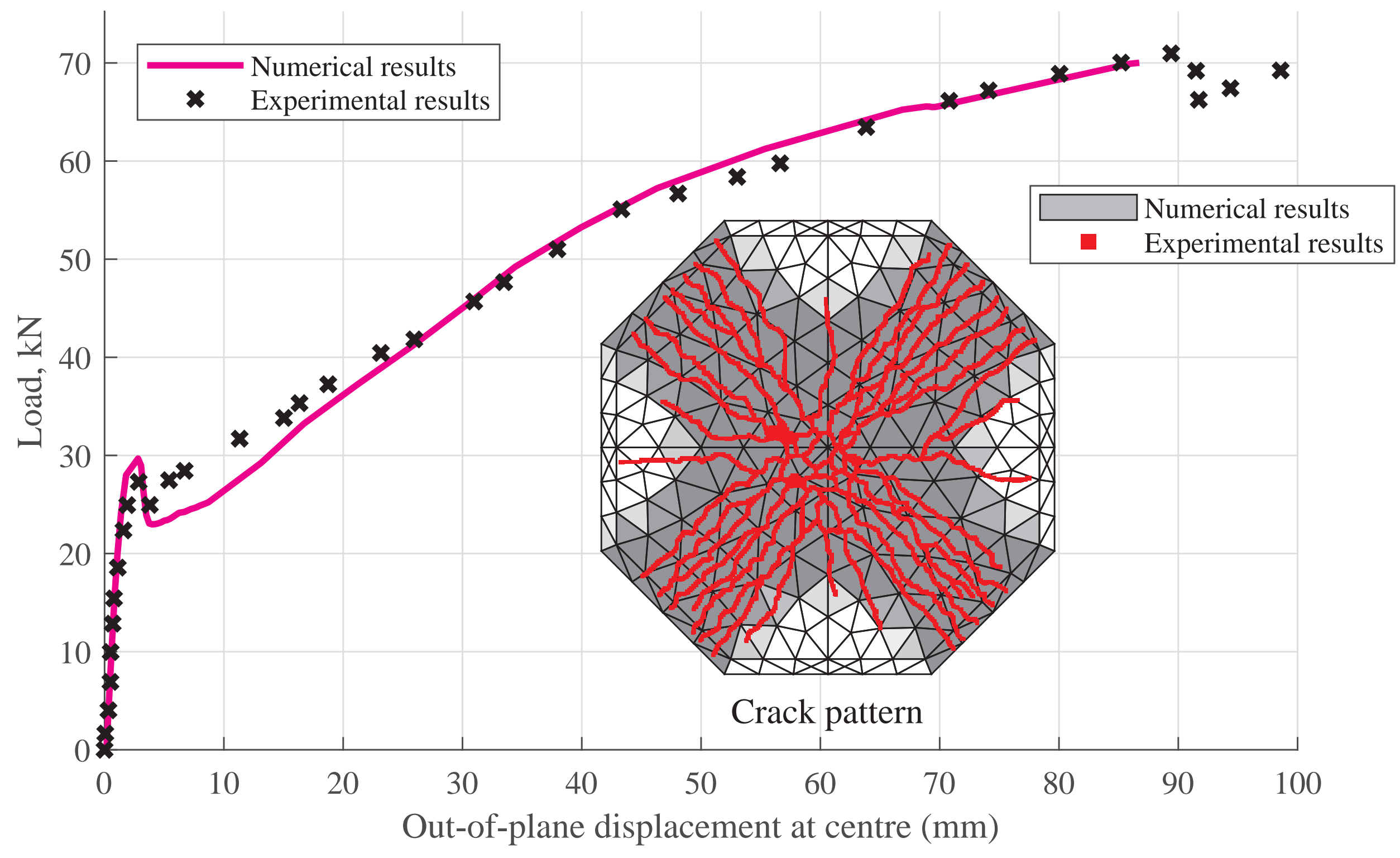


Figure 12. Circular slab experimental setup and employed finite element model
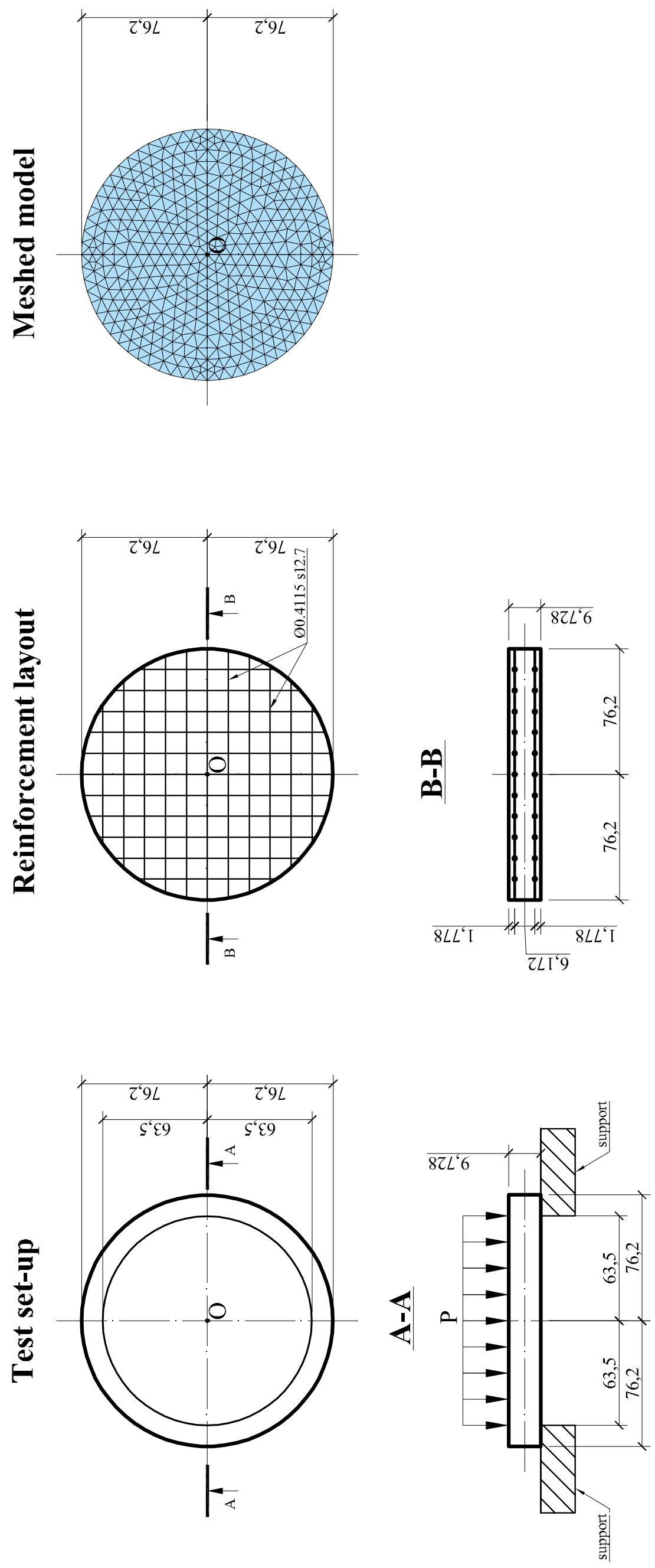


\begin{tabular}{|c|c|c|}
\hline & Octagonal slab & Circular slab \\
\hline \multicolumn{3}{|c|}{ Concrete material properties } \\
\hline Young's Modulus, N/mm ${ }^{2}$ & 31730 & 21752 \\
\hline Poison's Ratio & 0.2 & 0.2 \\
\hline Compressive strength, $\mathrm{N} / \mathrm{mm}^{2}$ & 50.9 & 28.68 \\
\hline Tensile strength, $\mathrm{N} / \mathrm{mm}^{2}$ & 2.7 & 1.45 \\
\hline Tensile softening modulus, $\mathrm{N} / \mathrm{mm}^{2}$ & 3520 & 1485.7 \\
\hline Pseudo-elastic range & 0.4 & 0.4 \\
\hline Factor for biaxial compressive interaction & 0.6 & 0.6 \\
\hline Elastic shear retention factor & 0.4 & 0.4 \\
\hline Factor scaling direct tensile stresses for shear interaction & 0.5 & 0.5 \\
\hline \multicolumn{3}{|c|}{ Steel material properties } \\
\hline Young's Modulus, N/mm² & 210000 & 199948 \\
\hline Yield stress, $\mathrm{N} / \mathrm{mm}^{2}$ & 550 & 303 \\
\hline Ultimate stress, $\mathrm{N} / \mathrm{mm}^{2}$ & 666 & 352 \\
\hline
\end{tabular}




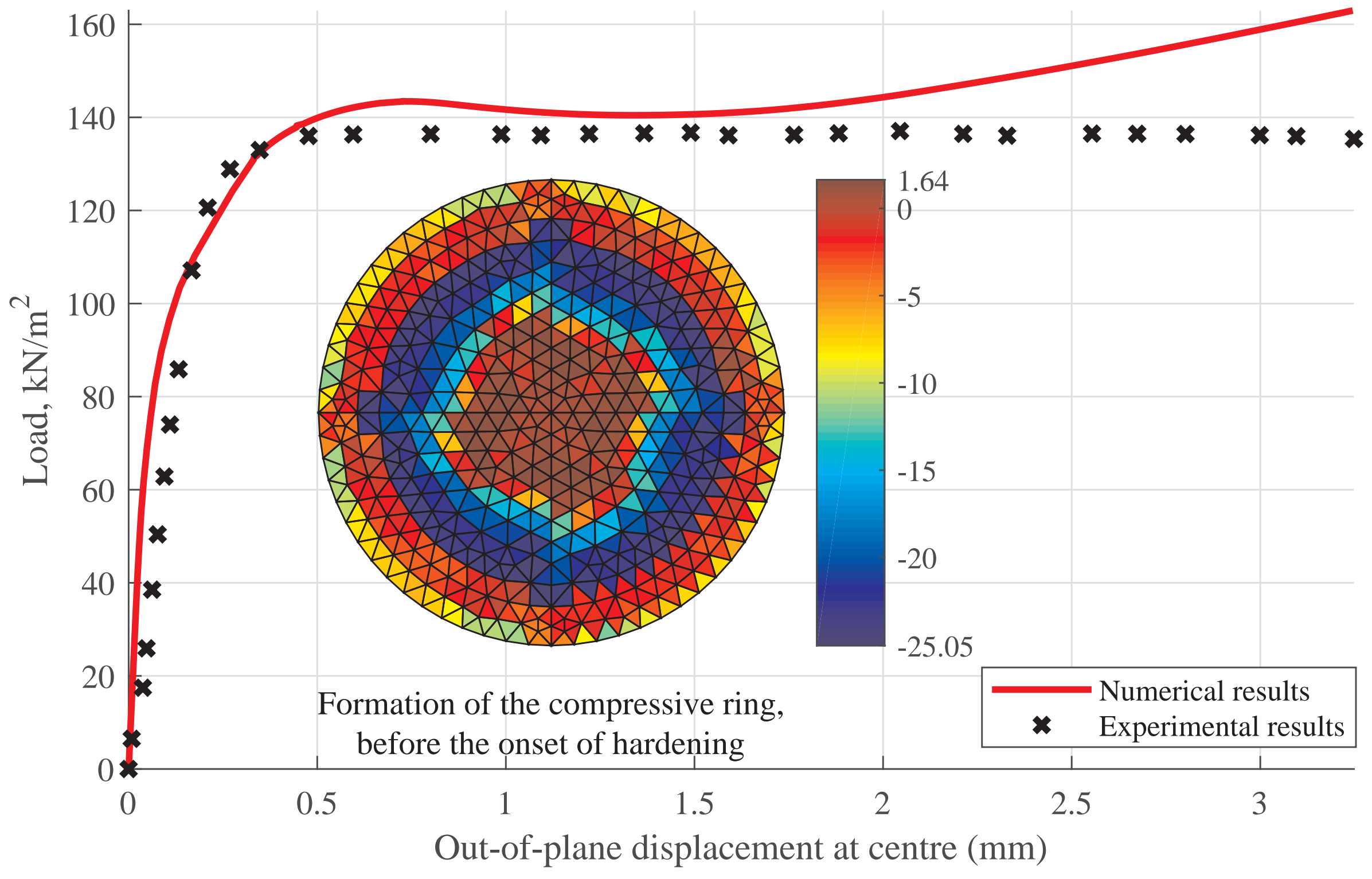




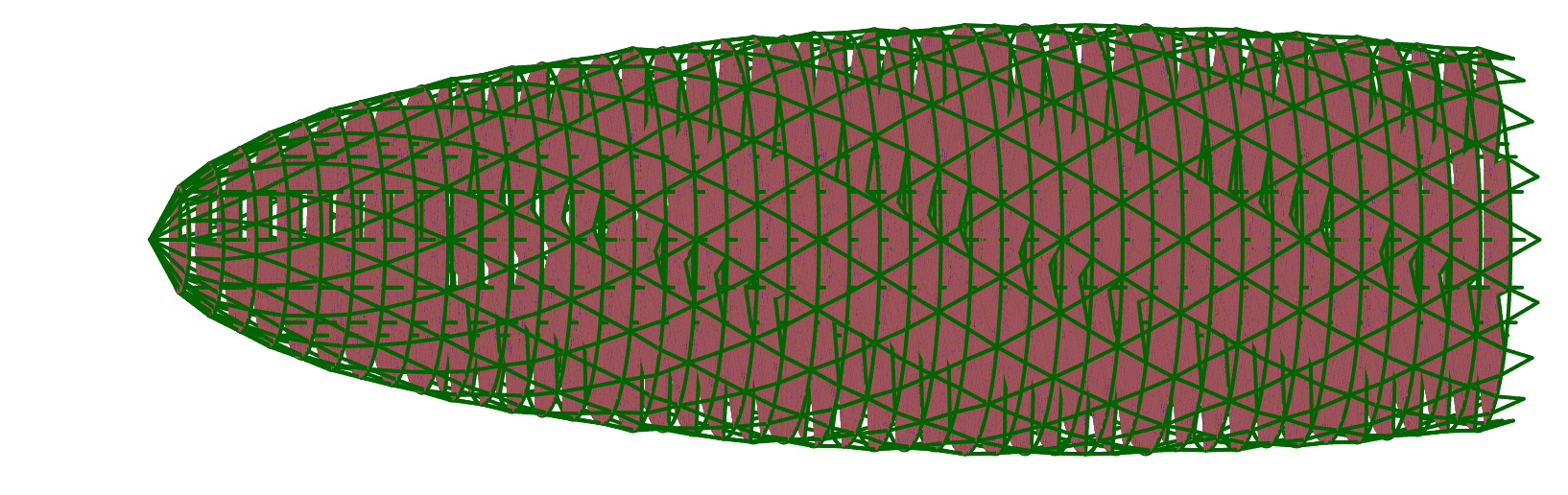

Figure 14. 3-D view of analysed structure

\section{Figure 14. 3-D view of analysed structure}
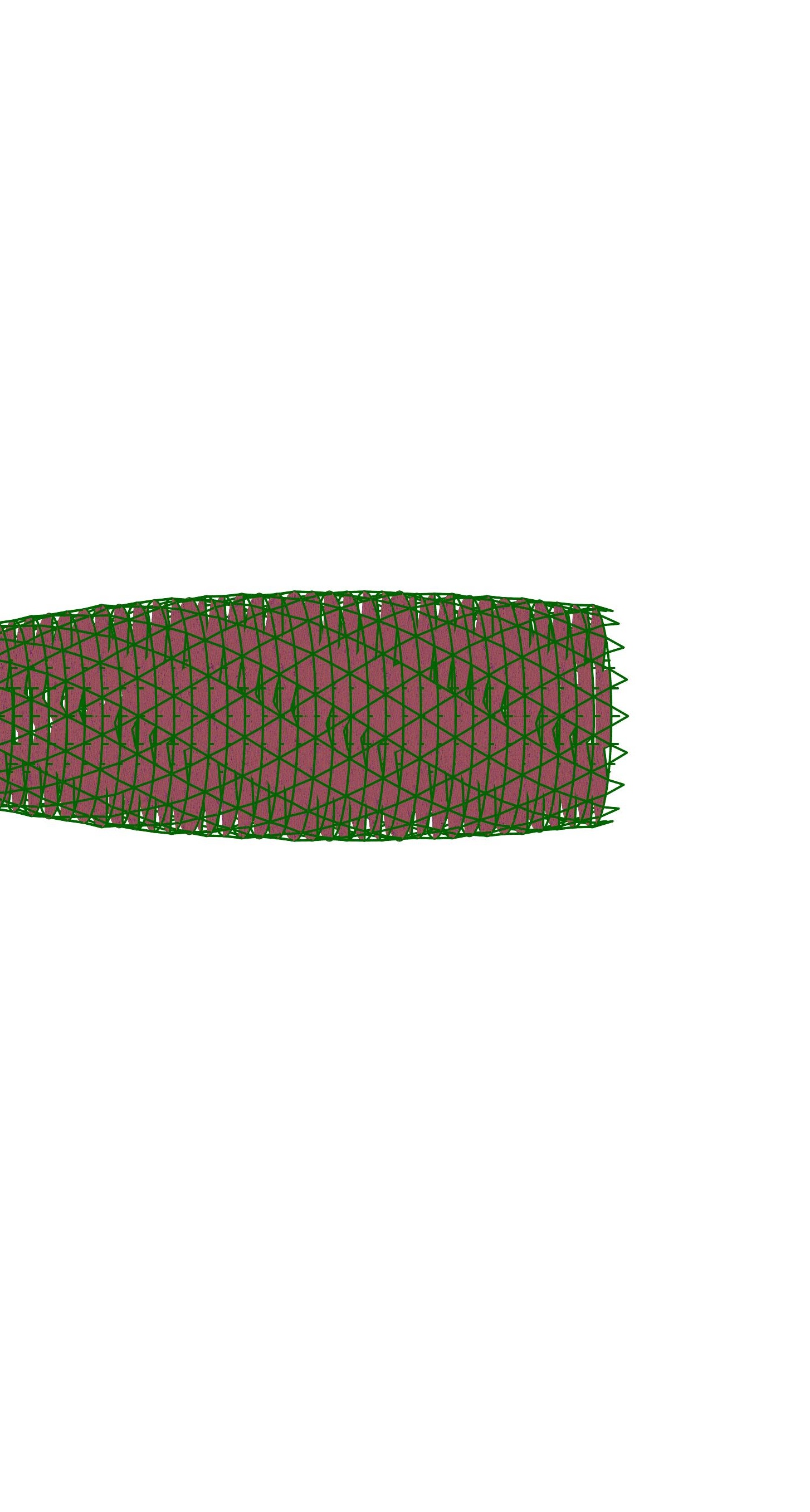
Figure 15. Irregular structure meshed floor plan

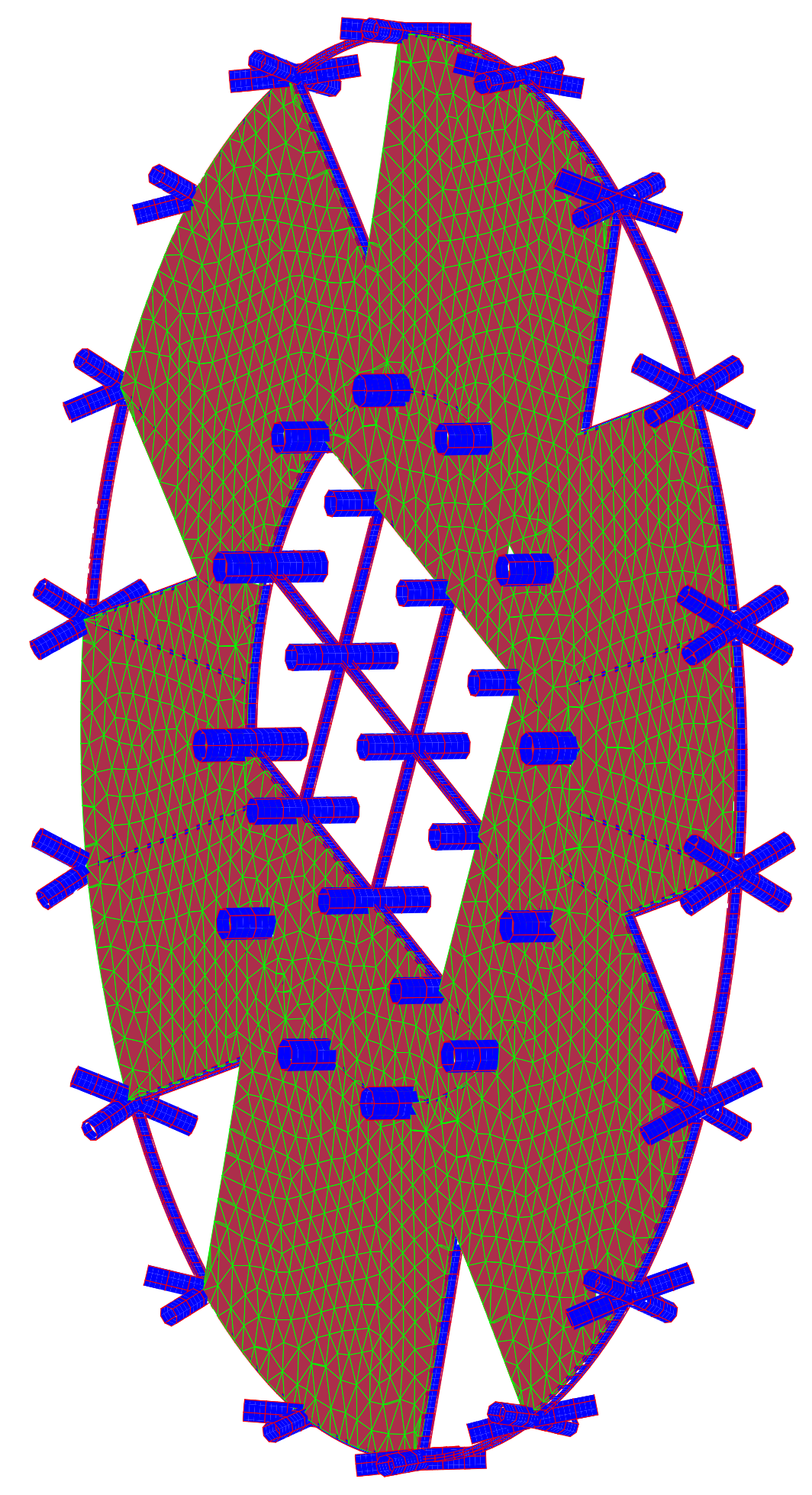


Figure 16. Slab deflections for the affected bay for the case of removal of two neighbouring internal columns
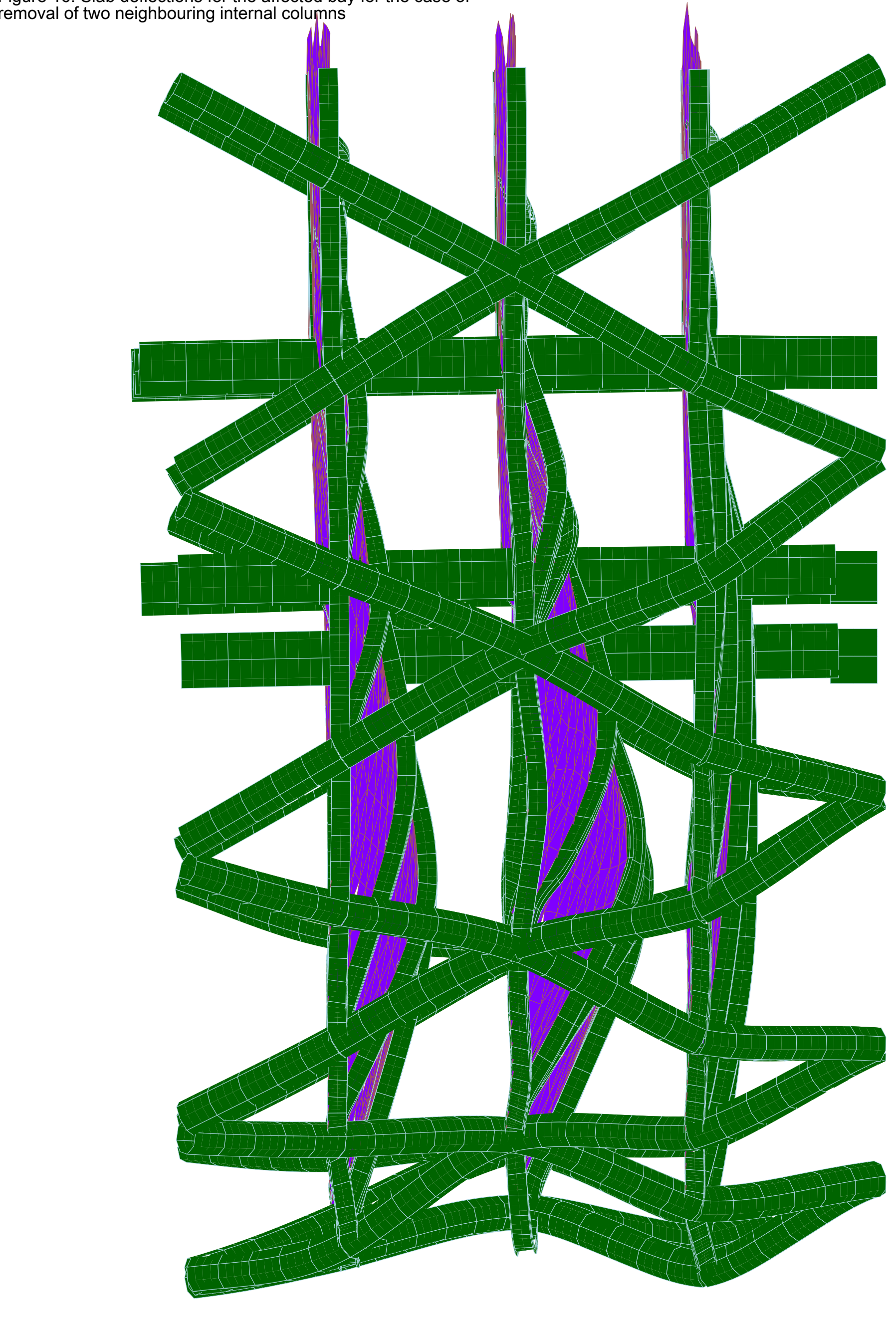
Click here to access/download Supplementary data jnlcopyright.pdf 